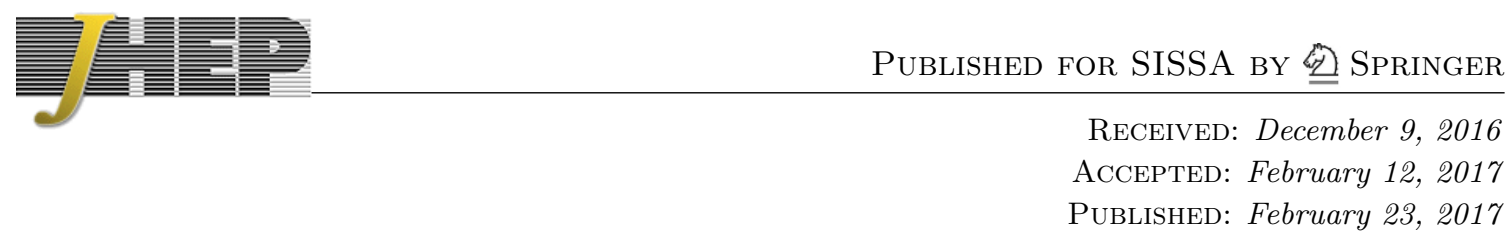

\title{
Solution of quantum integrable systems from quiver gauge theories
}

\author{
Nick Dorey ${ }^{a}$ and Peng Zhao ${ }^{b}$ \\ ${ }^{a}$ Department of Applied Mathematics and Theoretical Physics, University of Cambridge, \\ Cambridge, U.K. \\ ${ }^{b}$ Simons Center for Geometry and Physics, Stony Brook University, \\ Stony Brook, U.S.A. \\ E-mail: n.dorey@damtp.cam.ac.uk, pzhao@scgp.stonybrook.edu
}

ABSTRACT: We construct new integrable systems describing particles with internal spin from four-dimensional $\mathcal{N}=2$ quiver gauge theories. The models can be quantized and solved exactly using the quantum inverse scattering method and also using the Bethe/Gauge correspondence.

KEYwords: Bethe Ansatz, Supersymmetric gauge theory

ARXIV EPRINT: 1512.09367 


\section{Contents}

1 Introduction 1

2 Integrable systems from elliptic quiver gauge theories 5

2.1 The brane setup 5

2.2 Classical integrable systems from compactified gauge theories 9

2.3 The inhomogeneous spin Calogero-Moser model and its degenerate limits 12

3 Solution by the quantum inverse scattering method $\quad 14$

$\begin{array}{ll}3.1 & \text { The spin Calogero-Moser model } \\ \end{array}$

$\begin{array}{ll}3.2 & \text { The Hubbard-Toda model } \\ \end{array}$

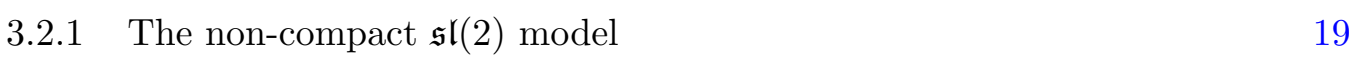

3.2.2 Solving the matrix Schrödinger equation 22

$\begin{array}{ll}3.2 .3 & \text { The compact } \mathfrak{s u}(2) \text { model }\end{array}$

4 Solution by the Bethe/Gauge correspondence 25

A The Inozemtsev limit to the Hubbard-Toda model 33

B Proof of classical integrability $\quad 34$

\section{Introduction}

The mysterious connections between integrable systems and supersymmetric gauge theories have lead to a fruitful interplay between the two subjects. One of the best-known examples is the relationship between classical integrable systems and four-dimensional $\mathcal{N}=2$ supersymmetric theories [1-3]. The Seiberg-Witten curve encoding the low-energy dynamics of the gauge theory coincides with the spectral curve encoding the mutually-commuting Hamiltonians of the integrable system. This coincidence has far-reaching consequences. Most importantly, it opens the door for studying the long-standing problem of the quantization of the Seiberg-Witten solution from the quantization of the corresponding integrable system, and vice versa.

In the last few years, this connection has been made more precise by the work of Nekrasov and Shatashvili [4-7]. They observed that quantization is related to deforming the theory by an $\Omega$-background in a two-dimensional plane. The rotation parameter $\epsilon$ in this plane is identified with Planck's constant $\hbar$. The supersymmetric vacua of the $\mathcal{N}=2$ theory are in one-to-one correspondence with the eigenstates of the quantum integrable system labeled by the solutions of the Bethe ansatz. The so-called Bethe/Gauge correspondence 
has given us new insights into dualities and symmetries between gauge theories [8-21]. ${ }^{1}$ For example, it has been used to establish new $2 \mathrm{~d} / 4 \mathrm{~d}$ dualities $[23,24]$ and $3 \mathrm{~d} / 5 \mathrm{~d}$ dualities $[25-$ 27], to shed light on 2d Seiberg-like dualities [28, 29], and 3d mirror symmetry [30]. The correspondence also solves quantum integrable models in finite volume, as it gives rise to thermodynamic Bethe ansatz equations by summing the instantons [6, 31-34].

Moreover, it has been conjectured that the supersymmetric vacua of any $\mathcal{N}=2$ theory in the Nekrasov-Shatashvili background corresponds to the solution of a quantum integrable system. Therefore finding the gauge-theoretic "dual" of a given classical integrable system will establish integrability at the quantum level. In the other direction, systematically identifying the integrable model "dual" to a given gauge theory is an intriguing open problem.

In this paper, we use the Bethe/Gauge correspondence to quantize and solve a new class of integrable systems arising from $4 \mathrm{~d} \mathcal{N}=2$ elliptic quiver gauge theories. ${ }^{2}$ The Coulomb branches of the gauge theories can be described as algebraic integrable systems with commuting Hamiltonians parametrized by a set of holomorphic coordinates constructed from the hyper-Kähler quotient. Real integrable systems arise on taking an appropriate middledimensional real section of the complex phase space. A very general class of integrable systems can be engineered this way describing particles with internal degrees of freedom. This class contains many well-known integrable systems such as the Calogero-Moser model and the Heisenberg spin chain in special corners of the parameter space.

Here we will consider two models, one of which is well-known. Both correspond to systems of $K$ particles moving in one dimension subject to periodic boundary conditions. We will denote the (real) positions and conjugate momenta of the particles as $x_{k}$ and $p_{k}$ respectively, $k=1, \ldots, K$. Each particle carries internal degrees of freedom corresponding to $N$ harmonic oscillators. For the $k$-th particle we have annihilation and creation operators $Q_{k}^{\alpha}, \tilde{Q}_{k}^{\alpha}$ with $\alpha=1, \ldots, N$. Both models have an internal symmetry group of rank $N-1$ corresponding to this index. In the classical version of each model, the variables described above obey canonical Poisson brackets. Using standard techniques from the theory of integrable systems we will construct quantum systems in which the corresponding operators obey canonical commutation relations.

The first model we consider is the elliptic spin Calogero-Moser model. In this case the particles carry classical $\mathfrak{s l}(N)$ "spins" which are constructed from the oscillators in the standard way,

$$
S_{k}^{\alpha \beta}=Q_{k}^{\alpha} \tilde{Q}_{k}^{\beta}-\frac{\delta^{\alpha \beta}}{N} \sum_{\gamma=1}^{N} Q_{k}^{\gamma} \tilde{Q}_{k}^{\gamma} .
$$

The classical Hamiltonian is given as,

$$
H=\sum_{k=1}^{K} \frac{p_{k}^{2}}{2}+\sum_{\ell>k}^{K} \sum_{\alpha, \beta=1}^{N} S_{k}^{\alpha \beta} S_{\ell}^{\beta \alpha} \wp\left(x_{k}-x_{\ell}\right),
$$

\footnotetext{
${ }^{1}$ See [22] for a pedagogical introduction to the Bethe/Gauge correspondence.

${ }^{2}$ The Seiberg-Witten geometry of this class of quiver gauge theories have recently been studied in [35, 36].
} 
where $\wp(z)$ is the Weierstraß elliptic function defined on a torus of complex structure $\tau$. The periodicity of this function for real arguments yields a system of particles moving in a box of size $L \sim \operatorname{Im} \tau$ subject to periodic boundary conditions.

As we review below, the classical model arises as a particular real section of the Coulomb branch of an $\hat{A}_{N-1}$ quiver gauge theory with gauge group $G=\mathrm{U}(1) \times \mathrm{SU}(K)^{N}$. The parameter $\tau$ corresponds to the complexified gauge coupling of the diagonal $\mathrm{U}(K)$ subgroup of $G$. The off-diagonal gauge couplings are tuned to a particular strong-coupling point where a hidden global $A_{N-1}$ symmetry appears. Following the recipe introduced by Nekrasov and Shatashvili, quantization is achieved by introducing an $\Omega$-background in one plane. The induced twisted superpotential of the resulting $2 \mathrm{~d}$ effective theory corresponds to the Yang-Yang potential which determines the spectrum of the corresponding quantum integrable system. To select the real section corresponding to the spin Calogero-Moser model, it is also necessary to choose an appropriate electro-magnetic duality frame for the quiver gauge theory. This point is discussed further in section 4 below.

In principle, with the above identification, the Nekrasov-Shatashvili procedure provides a quantization of the model for all values of the parameters. Here, we will focus on the large-volume limit $L \sim \operatorname{Im} \tau \gg 1$, where the system can also be solved using the asymptotic Bethe ansatz. The idea of the asymptotic Bethe ansatz is to first solve the problem in the limiting case $\operatorname{Im} \tau=\infty$ where the particles move on an infinite line, with the $k$-th and the $\ell$-th particles interacting via the two-body potential,

$$
V\left(x_{k}-x_{\ell}\right) \sim \sum_{\alpha, \beta=1}^{N} \frac{S_{k}^{\alpha \beta} S_{\ell}^{\beta \alpha}}{4 \sinh ^{2}\left(\frac{x_{k}-x_{\ell}}{2}\right)} .
$$

This gives rise to a scattering problem for asymptotic states corresponding to free particles carrying classical spins $S_{k}^{\alpha \beta}$. For these asymptotic states, quantization proceeds in a straightforward way by promoting the canonical Poisson brackets of the variables $\left\{x_{k}, p_{k}, Q_{k}^{\alpha}, \tilde{Q}_{k}^{\alpha}\right\}$ to canonical commutation relations. For appropriate values of the conserved quantities, the resulting spin operators $\hat{S}_{k}^{\alpha \beta}$ act in lowest-weight irreducible representations of $\mathfrak{s l}(N, \mathbb{R})$. At least for $L \sim \operatorname{Im} \tau \gg 1$, the quantum model can be thought of as a system of $K$ particles each carrying a non-compact "spin" corresponding to a lowest-weight representation of $\mathfrak{s l}(N, \mathbb{R})$.

Quantum integrability of the model requires that multi-particle scattering factorizes into a product of successive two-body scattering processes. Furthermore, the consistency of factorized scattering requires that the two-body S-matrix obey the Yang-Baxter equation. Our approach here, will be to assume factorization of multi-particle scattering. However, we will check the Yang-Baxter equation explicitly. The first step in the analysis is to solve the Schrödinger equation describing the scattering of two of these particles. As advertised, the resulting two-body S-matrix indeed obeys the Yang-Baxter equation. Through our assumption of factorization, the multi-particle S-matrix is then determined. We find that it can be diagonalized explicitly using the quantum inverse scattering method. The last step is to impose periodic boundary conditions on the resulting scattering wave functions which leads to the asymptotic Bethe ansatz equations. The energy spectrum of the model 
is then determined by solutions of these equations. Our main result is that the NekrasovShatashvili quantization procedure applied to the quiver gauge theory, yields the same Bethe ansatz equations and therefore the same spectrum.

The second model we study involves a different limit of the parameters of the full inhomogeneous system. For the original elliptic Calogero-Moser model for $K$ particles without spin, with Hamiltonian,

$$
H=\sum_{k=1}^{K} \frac{p_{k}^{2}}{2}+m \sum_{\ell>k}^{K} \wp\left(x_{k}-x_{\ell}\right)
$$

There is a well-known limit, first discussed by Inozemtsev [37], which yields the $K$-body Toda chain with Hamiltonian,

$$
H^{\text {Toda }}=\sum_{k=1}^{K} \frac{p_{k}^{2}}{2}+\sum_{k=1}^{K-1} e^{X_{k}-X_{k+1}}+\Lambda^{2 K} e^{X_{K}-X_{1}}
$$

where $\Lambda=m \exp (2 \pi i \tau / K)$. In the classical version of the correspondence to supersymmetric gauge theory, the scalar elliptic model corresponds to the $\mathcal{N}=2$ super Yang-Mills theory with an adjoint hypermultiplet of mass $m$ and complexified coupling $\tau$ (also known as the $\mathcal{N}=2^{*}$ theory). The Inozemtsev limit coincides with the standard decoupling limit for the adjoint hypermultiplet which yields the minimal $\mathcal{N}=2$ gauge theory. The latter is asymptotically free and is characterized by the RG-invariant scale $\Lambda=m \exp (2 \pi i \tau / K)$.

Here, we will take a similar limit for the elliptic quiver gauge theory which yields a Toda-like chain for particles with internal degrees of freedom. As before we have $K$ particles moving in one dimension with positions $x_{k}$ and momenta $p_{k}, k=1, \ldots, K$, each particle having $N$ internal harmonic oscillator degrees of freedom with annihilation and creation operators $Q_{k}^{\alpha}, \tilde{Q}_{k}^{\alpha}$ for $\alpha=1, \ldots, N$. Now we form $\mathfrak{s l}(N)$-invariant hopping operators between the $k$-th and the $\ell$-th sites,

$$
A_{k \ell}=\sum_{\alpha=1}^{N} Q_{k}^{\alpha} \tilde{Q}_{\ell}^{\alpha}
$$

By taking an Inozemtsev-like limit, we find a classical integrable system with quadratic Hamiltonian,

$$
H^{\mathrm{HT}}=H^{\mathrm{Toda}}+\sum_{k=1}^{K} \frac{A_{k k}^{2}}{4}+\frac{1}{2}\left[\sum_{k=1}^{K-1} e^{\frac{x_{k}-X_{k+1}}{2}}\left(A_{(k+1) k}+A_{k(k+1)}\right)+\Lambda^{K} e^{\frac{X_{K}-X_{1}}{2}}\left(A_{1 K}+A_{K 1}\right)\right] .
$$

We check directly the classical integrability of this model.

As above we study the quantization of the above system in the framework of the asymptotic Bethe ansatz, which gives an accurate description of the system in the limit of large volume. In this case, the quantum system consists of $K$ particles, interacting via exponential potentials, each carrying $N$ harmonic oscillator degrees of freedom. The corresponding occupation numbers are individually conserved when the particles are far 
apart. However, the interaction terms in the Hamiltonian proportional to $A_{k(k+1)}$, mean that occupation number can be transferred from one particle to the next one in the chain. Freezing the positions of the $K$ particles, the resulting dynamics of the oscillator degrees of freedom is closely related to the Hubbard model. For this reason we propose to call the system (1.7), the Hubbard-Toda chain. Once again our main result is a comparison of the large-volume solution of the model via the asymptotic Bethe ansatz with the appropriate application of the Bethe/Gauge correspondence, which yields exact agreement.

The Bethe/Gauge correspondence not only provides a quantization of the corresponding classical integrable system, but also provides the solution to the full quantum problem. Since the Bethe ansatz equations are mapped directly to the supersymmetric vacua of the quiver gauge theory, the vacuum equations provide a prediction for the scalar part of the Smatrix. The prediction agrees perfectly with the direct solutions of the matrix Schrödinger equation. Furthermore, the instanton partition function yields a set of thermodynamic Bethe ansatz equations that determine the finite-size spectrum of the model.

This paper is organized as follows. In section 2, we describe the brane setup of the elliptic quiver and how classical integrable systems arise from the Coulomb and the Higgs branch descriptions. In section 3, we quantize the integrable system and exactly solve the system using the quantum inverse scattering method. In section 4, we use the Bethe/Gauge correspondence to predict the scalar part of the S-matrix. The appendices contain details on the Inozemtsev limit and the classical integrability of the Hubbard-Toda model.

\section{Integrable systems from elliptic quiver gauge theories}

\subsection{The brane setup}

We consider $4 \mathrm{~d} \mathcal{N}=2$ quiver gauge theories whose quiver diagram is the affine Dynkin diagram of $\hat{A}_{N-1}$ type. The gauge group is $\mathrm{U}(1)_{D} \times \mathrm{SU}(K)^{N}$. There is a vector multiplet for each $\mathrm{SU}(K)$ factor and a bi-fundamental hypermultiplet for adjacent $\mathrm{SU}(K)$ factors. Each gauge group factor has a gauge coupling $g_{\alpha}$. The $\beta$ function vanishes and the theories are conformal. We combine the gauge coupling and the theta angle into a marginal gauge coupling $\tau_{\alpha}=4 \pi i / g_{\alpha}^{2}+\vartheta_{\alpha} / 2 \pi$. The theories at low energy have a moduli space of vacua known as the Coulomb and the Higgs branches. In the Coulomb branch, the complex scalars in the vector multiplet acquire vacuum expectation values and the gauge group is broken down to its Cartan subgroup. In the Higgs branch, the complex scalars in the hypermultiplet acquire vacuum expectation values and break the gauge group completely.

The quiver gauge theories can be embedded in string theory as the world-volume theories of $K$ D4-branes intersecting $N$ NS5-branes in the Type IIA string theory. The D4-branes have world volume in the 01236 direction and is compactified in the $x^{6}$ direction. The NS5-branes have world volume in the 012345 direction. This setup is called the elliptic model as it arises from M-theory, which has an additional compact $x^{10}$ direction [38]. We will refer to them as elliptic quiver theories to distinguish from the corresponding elliptic integrable models.

The generic brane configuration is shown in figure 1 . The brane setup preserves eight real supercharges and engineers a $4 \mathrm{~d} \mathcal{N}=2$ gauge theory with gauge group $\mathrm{U}(1)_{D} \times$ 


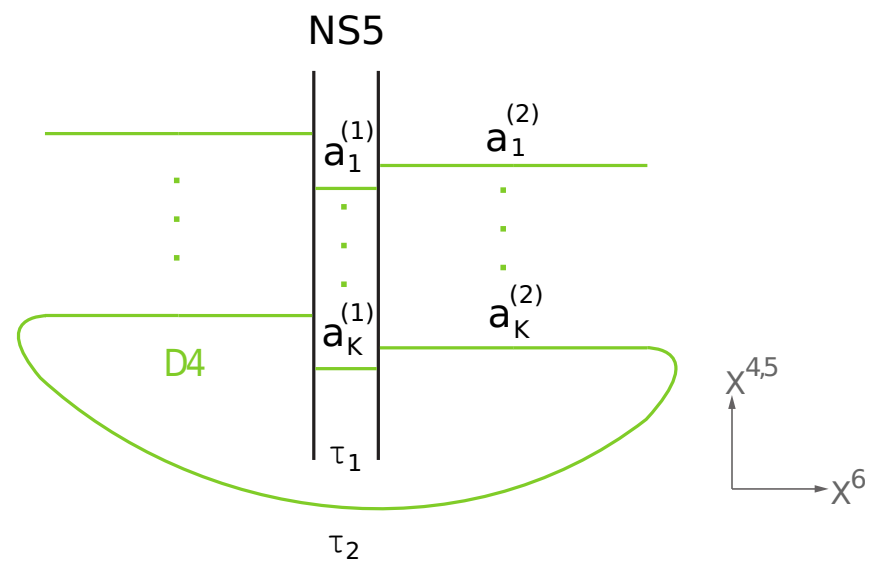

Figure 1. The Type IIA brane construction of the $\mathrm{U}(1)_{D} \times \mathrm{SU}(K)^{2}$ quiver gauge theory.

$\mathrm{SU}(K)^{N}$. Throughout the paper, we will use $\alpha, \beta=1, \ldots, N$ to denote $\operatorname{SL}(N)$ indices, and $k, \ell=1, \ldots, K$ to denote $\mathrm{SU}(K)$ indices. The positions $\left(a_{1}^{(\alpha)}, \ldots, a_{K}^{(\alpha)}\right)$ of the D4-branes between the $\alpha$-th and the $(\alpha+1)$-th NS5-branes label the Coulomb branch moduli of the $\alpha$-th $\mathrm{SU}(K)$ factor of the gauge group. The diagonal $\mathrm{U}(1)_{D}$ factor corresponds to the center-of-mass position of all the D4-branes and decouples from the low-energy dynamics. The relative center-of-mass positions between the neighboring D4-branes define the mass of the bi-fundamental hypermultiplets $m_{\alpha}$. The mass can be arbitrarily chosen by imposing a twisted periodicity condition on the $x^{6}$ circle: $a_{k}^{(\alpha+N)}=a_{k}^{(\alpha)}+m$ as $x^{6} \rightarrow x^{6}+2 \pi R_{6}$ such that $\sum_{\alpha} m_{\alpha}=m$. Because we consider an equal number of D4-branes on either side of the NS5-brane, the theory is conformal. The separation of the NS5-branes in the $x^{6}$ and the $x^{10}$ directions are proportional to the gauge coupling $1 / g_{\alpha}^{2}$ and the theta angle $\vartheta_{\alpha}$, respectively. The gauge coupling $1 / g^{2}=\sum_{\alpha} 1 / g_{\alpha}^{2}$ of the diagonal subgroup $\mathrm{U}(K)=\mathrm{U}(1)_{D} \times \mathrm{SU}(K) / \mathbb{Z}_{K}$ is proportional to the radius $R_{6}$ of the $x^{6}$ circle.

In the limit when one of the gauge couplings becomes weakly coupled, the corresponding gauge group can be frozen to become a global symmetry. The dynamical D4branes parametrizing the Coulomb branch moduli become rigid D4-branes labeling the flavor charges. The elliptic quiver then reduces to a linear quiver. Conversely, the elliptic quiver can be obtained from the linear quiver by weakly gauging the global symmetry. As we will see, these have clear analogues on the integrable systems side where the weakcoupling limit corresponds to taking the infinite-volume limit. The two-body S-matrix is well-defined and can be solved for on the infinite line. We then pass to a large circle and use the asymptotic Bethe ansatz to determine the spectrum of the system.

There is a special point in the Coulomb branch moduli space where $a_{k}^{(\alpha)}=a_{k}^{(\alpha+1)}$ for all $k$ and for $\alpha=1, \ldots, N-1$. At this point, the D4-branes on either side of the $N-1$ NS5branes coincide and reconnect. The $N-1$ NS5-branes can then be lifted in the orthogonal $x^{7}$ direction and the $4 \mathrm{~d}$ theory moves onto its Higgs branch. The special point at which the Coulomb and the Higgs branch meet is called the Higgs branch root, as shown on the left of figure 2. The separation of the NS5-branes in the $x^{7}$ direction corresponds to the Higgs branch vacuum expectations value. The theory in the Higgs branch admits vortex 

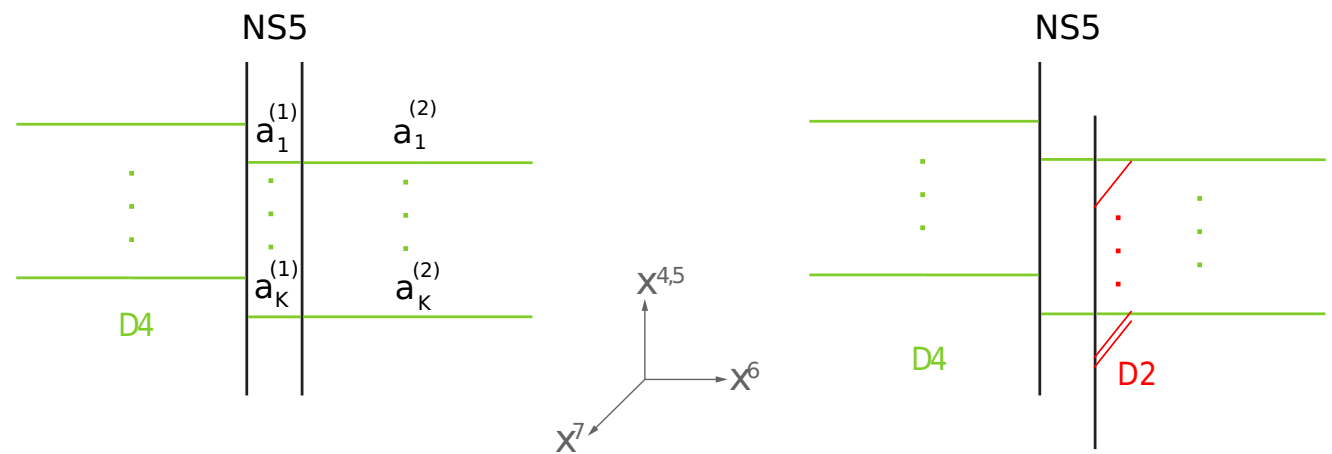

Figure 2. At the Higgs branch root, D4-branes on either side of an NS5-brane reconnect. We move onto the Higgs branch by lifting the NS5-brane in the $x^{7}$ direction. The coupled $2 \mathrm{~d}-4 \mathrm{~d}$ system describes vortex strings probing the $4 \mathrm{~d}$ theory.

string solutions. They appear as D2-branes stretched between the lifted NS5-branes and the D4-branes in the 0127 direction [39, 40], as shown on the right of figure 2 . The vortex string tension is proportional to the Higgs branch vacuum expectation value. The number of D2-branes $M_{\alpha}$ is arbitrary. The world-volume theory on the D2-branes is a $2 \mathrm{~d} \mathcal{N}=(2,2)$ gauged linear sigma model with gauge group $\mathrm{U}\left(M_{1}\right) \times \mathrm{U}\left(M_{1}+M_{2}\right) \times \cdots \times \mathrm{U}\left(M_{1}+\cdots+\right.$ $\left.M_{N-1}\right)$ [41]. The fundamental strings stretched between the D2 and the D4-branes define the fundamental and the anti-fundamental chiral multiplets. The separations of the NS5branes in the $x^{6}$ direction is the Fayet-Iliopoulos parameter $r_{\alpha}$ of the $2 \mathrm{~d}$ theory. It combines with the $2 \mathrm{~d}$ theta angle to form the complexified coupling $\hat{\tau}_{\alpha}=i r_{\alpha}+\theta_{\alpha} / 2 \pi$, which will be identified with the $4 \mathrm{~d}$ gauge coupling $\tau_{\alpha}$. When the Fayet-Iliopoulos parameter is turned off, the $2 \mathrm{~d}$ theory is in its Coulomb branch parametrized by the vacuum expectation values of the twisted chiral multiplet scalars $\sigma_{i}^{(\alpha)}$, which label the positions of the D2-branes in the $x^{4}+i x^{5}$ plane.

Surprisingly, the $2 \mathrm{~d}$ theory captures the physics of the $4 \mathrm{~d}$ theory. This was first suggested by matching the BPS spectra of the two theories $[42,43]$. This $2 \mathrm{~d} / 4 \mathrm{~d}$ duality was made more precise when the $4 \mathrm{~d}$ theory is subject to the $\Omega$-background in the NekrasovShatashvili limit $[23,24]$. In this deformed background, the $4 \mathrm{~d}$ theory is localized onto a $2 \mathrm{~d}$ subspace preserving $\mathcal{N}=(2,2)$ supersymmetry. The theory is described by an effective twisted superpotential, which coincides with that of the theory living on the vortex string.

We will be interested in two special configurations and their decoupling limits.

1. We take the limit that the diagonal $\mathrm{U}(1)_{D} \times \mathrm{SU}(K)$ becomes weakly coupled with gauge couplings $1 / g_{\alpha}, \alpha=1, \ldots, N-1$ held fixed. This implies that $g \rightarrow 0$ and hence $g_{N} \rightarrow 0$. In this limit, the theory factorizes into a diagonal $\mathrm{U}(1)_{D} \times \mathrm{SU}(K)$ gauge group and a linear quiver with gauge group $\mathrm{SU}(K)^{N-1}$. The diagonal gauge group has an adjoint hypermultiplet of mass $m$, while the linear quiver has $K$ fundamental hypermultiplets and $K$ anti-fundamental hypermultiplets. The Coulomb branch moduli of the diagonal gauge group appear as mass parameters for the hypermultiplets in the linear quiver. The non-trivial periodicity condition defines the adjoint hypermultiplet mass $m$. For the $\mathrm{U}(1)_{D} \times \mathrm{SU}(K)^{2}$ theory shown in figure 1 , this 

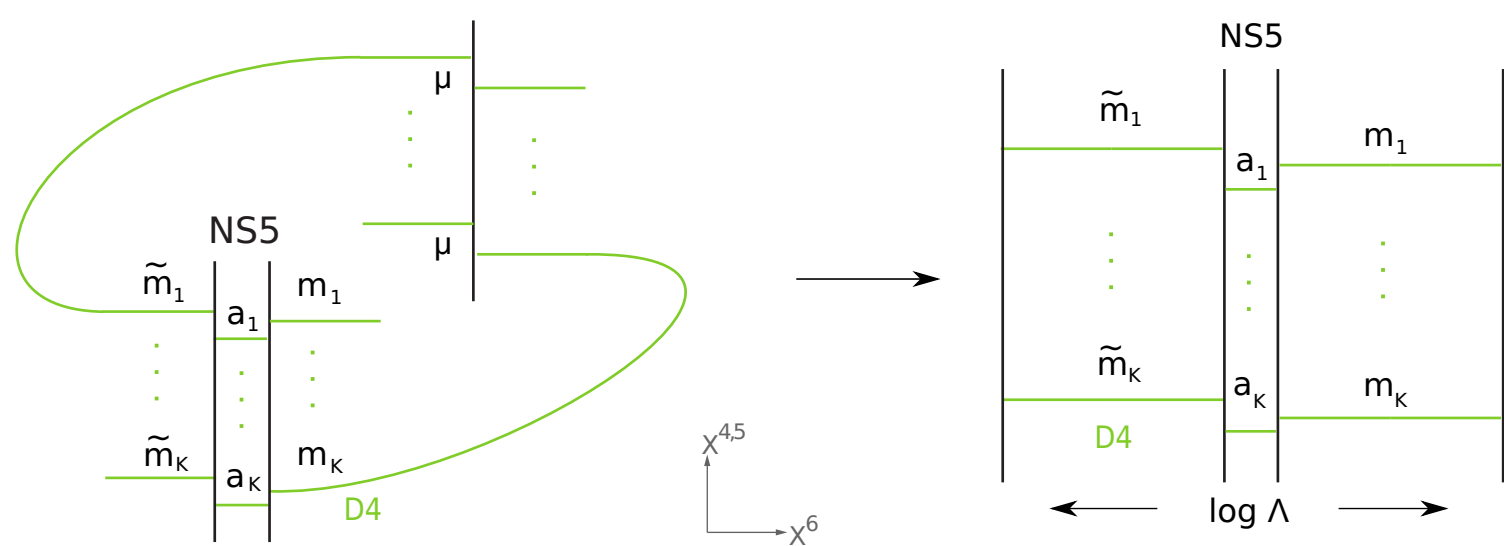

Figure 3. Flowing from an $\mathcal{N}=2^{*}$ theory to a pure $\mathcal{N}=2$ theory: we take the weak-coupling limit and keep the combination $\Lambda^{K}=\mu^{K} e^{2 \pi i \tau_{N}}$ fixed.

corresponds to taking $\operatorname{Im} \tau_{1} \rightarrow 0$ while sending $\operatorname{Im} \tau_{2} \rightarrow \infty$. In terms of integrable systems, this is the large-volume limit of the elliptic spin Calogero-Moser model.

2. As before we take the diagonal coupling to zero but now we send $g_{1}=g_{N} \rightarrow 0$ such that the gauge group factorizes into a weakly-coupled $\mathrm{SU}(K) \times \mathrm{SU}(K)$ and an $\mathrm{SU}(K)^{N-2}$ linear quiver. The $\mathrm{SU}(K) \times \mathrm{SU}(K)$ has a bi-fundamental hypermultiplet of mass $\mu=m_{N}$ and a bi-fundamental hypermultiplet of mass $\sum_{\alpha=1}^{N-1} m_{\alpha}$. The Coulomb branch moduli of the $\mathrm{SU}(K) \times \mathrm{SU}(K)$ gauge group appear as mass parameters for the fundamental and the anti-fundamental hypermultiplets. We take the limit where $\mu$ becomes infinitely massive while the combination $\Lambda^{K}=\mu^{K} e^{2 \pi i \tau_{N}}$ is fixed. For a single gauge group factor, this corresponds to flowing from an $\mathcal{N}=2^{*}$ theory to a pure $\mathcal{N}=$ 2 theory by decoupling the adjoint hypermultiplet while taking the weak-coupling limit such that a dynamical scale $\Lambda$ is generated via dimensional transmutation.

In the brane picture, we take a single NS5-brane to be at half-period. This is depicted in the left of figure 3. In our limit, the half-period $i \pi \tau_{N}$ and the separation $\mu$ of the D4-branes ending on the single NS5-brane are sent to infinity. The NS5-brane effectively becomes two disjoint NS5-branes separated by a distance $\log \Lambda$ and each sourcing $K$ semi-infinite D4-branes ending on the stack of NS5-branes, as shown on the right of figure 3. As seen from the other $N-1$ NS5-branes, the two ends of the D4-branes are frozen and define a global symmetry group. The positions of these D4branes define the mass of the fundamental and the anti-fundamental hypermultiplets $m_{k}$ and $\tilde{m}_{k}$. The theory effectively reduces to a linear quiver gauge theory.

Each example corresponds to a classical integrable system. The Seiberg-Witten curve coincides with the spectral curve of each integrable system. The chiral ring corresponds to the conserved Hamiltonians. By matching the curves, we may identify the parameters. We summarize the dictionary between gauge theories and integrable systems in table 1 . In the next section, we examine in more detail how to obtain the integrable systems from the gauge theory data. 


\begin{tabular}{|c|c|}
\hline Gauge theory & Integrable system \\
\hline$N$ NS5-branes & $\mathfrak{s l}(N, \mathbb{C})$ spin \\
\hline$K$ D4-branes & $K$ particles \\
\hline$M$ D2-branes & $M$ magnons \\
\hline Marginal coupling $\tau$ & System size $L=\operatorname{Im} \tau$ \\
\hline Coulomb branch moduli $a_{k}$ & Particle momentum $p_{k}$ \\
\hline Twisted chiral multiplet scalar $\sigma_{i}$ & Magnon rapidity $\lambda_{i}$ \\
\hline Adjoint hypermultiplet mass $m$ & Interaction strength $m$ \\
\hline Fundamental hypermultiplet mass $m_{k}$ & Inhomogeneity and spin $p_{k}-s_{k} \epsilon$ \\
\hline Anti-fundamental hypermultiplet mass $\tilde{m}_{k}$ & Inhomogeneity and spin $p_{k}+s_{k} \epsilon$ \\
\hline$\Omega$-deformation parameter $\epsilon$ & Planck's constant $-i \hbar$ \\
\hline
\end{tabular}

Table 1. Dictionary between gauge theories and integrable systems.

\subsection{Classical integrable systems from compactified gauge theories}

The Coulomb branch of the elliptic quiver gauge theory is effectively an abelian theory with gauge group $\mathrm{U}(1)^{r}$, where $r=K N-N+1$. Its dynamics is governed by the pre-potential $\mathcal{F}$, which can be determined by the Seiberg-Witten curve $\Sigma$ and the meromorphic one-form $\lambda_{\mathrm{SW}}$. The Coulomb branch moduli space is parametrized by periods of the Seiberg-Witten differential around homology cycles $\left\{A_{I}, B_{I}\right\}$ for $I=1, \ldots, r$,

$$
\vec{a}=\frac{1}{2 \pi i} \oint_{\vec{A}} \lambda_{\mathrm{SW}}, \quad \vec{a}^{D}=\frac{1}{2 \pi i} \oint_{\vec{B}} \lambda_{\mathrm{SW}}
$$

The low-energy effective theory takes the form of a non-linear sigma model on the Coulomb branch whose metric can be read off from the period matrix

$$
\tau_{I J}=\frac{\partial \mathcal{F}}{\partial a^{I} \partial a^{J}} .
$$

This gives the Coulomb branch the structure of the base of a complex integrable system. The full structure of the Seiberg-Witten integrable system can be seen more clearly when we compactify the $4 \mathrm{~d}$ theory on a circle down to three dimensions [44]. One can turn on Wilson loops around the compactified circle, which define real periodic scalars $\theta_{e}^{I}=\oint A_{3}^{I} d x^{3}$. The dual photons define another set of real periodic scalars $\theta_{m}^{I}$. The $3 \mathrm{~d}$ Coulomb branch is parametrized by the complex scalars $a^{I}$ as well as $\theta_{e}^{I}$ and $\theta_{m}^{I}$. It takes the form of a Jacobian fibration over the base manifold where the Jacobian torus is parametrized by $z_{I}=\theta_{m, I}-\tau_{I J} \theta_{e}^{J}$. The variables $\left(a^{I}, z_{I}\right)$ play the role of the action-angle variables of the complex integrable system. The dynamics are linear flows on the Jacobian torus.

As suggested by Kapustin, the Coulomb branch of the compactified quiver gauge theory has an alternative description in terms of the Higgs branch of the mirror theory [45]. The Higgs branch does not receive quantum corrections and provides an alternative, simpler description of the theory. Let us review how to obtain the Higgs branch description of the 
theory via a sequence of string dualities. The branes are compactified in the $x^{3}-x^{6}$ torus. Performing a T-duality along the $x^{3}$ circle, the D4-branes become D3-branes along the 0126 direction. S-duality interchanges the NS5-branes with D5-branes along the 012345 direction. A further T-duality along the $x^{3}$ circle maps the D3-D5 system into a D4-D4' system with $K$ D4-branes wrapped around the $x^{3}-x^{6}$ torus and $N$ D4'-branes localized on the torus. This is the impurity theory [46].

Alternatively, we can T-dualize along the $x^{6}$ circle to obtain a D2-D6 system where the $K$ D2-branes can be interpreted as $\mathrm{SU}(N)$ instantons moving in the four transverse directions inside the D6-brane. Because the $x^{3}$ and the $x^{6}$ directions are compactified, the instantons live on $\mathbb{R}^{2} \times T^{2}$. We further turn on Wilson lines for the $\mathrm{SU}(N)$ gauge field on $T^{2}$, which specifies the positions of the impurities on the dual torus. The instanton moduli space describes the Higgs branch of the $\mathrm{SU}(K)$ gauge theory, which in turn coincides with the Coulomb branch of the original gauge theory via the mirror map [47]. ${ }^{3}$

This moduli space of $K \mathrm{SU}(N)$ instantons on $\mathbb{R}^{2} \times T^{2}$ can then be mapped to a $2 \mathrm{~d}$ theory living on the dual torus $\hat{T}^{2}$ using the Nahm transform. The $2 \mathrm{~d}$ theory is described by a complex $\mathrm{U}(K)$ gauge field $A_{z}(z, \bar{z}), A_{\bar{z}}(z, \bar{z})$ and an adjoint scalar $\phi(z, \bar{z})$. The fields have prescribed boundary conditions parametrized by the impurities $Q_{k}^{\alpha}, \tilde{Q}_{k}^{\alpha}$ in the fundamental and the anti-fundamental representations of $\mathrm{U}(K)$ at the punctures $z_{\alpha}$. The infinite-dimensional space spanned by these fields is endowed with a canonical symplectic form

$$
\omega_{\infty}=\int d^{2} z \sum_{k, \ell=1}^{K} d \phi_{k \ell}(z, \bar{z}) \wedge d A_{\ell k}^{z}(z, \bar{z})+\sum_{k=1}^{K} \sum_{\alpha=1}^{N} d Q_{k}^{\alpha} \wedge d \tilde{Q}_{k}^{\alpha} .
$$

The F-term equations modulo the complexified gauge symmetry $\mathrm{U}(K, \mathbb{C})$ give rise to the Hitchin equations

$$
\partial_{\bar{z}} \phi_{k \ell}+\left[A_{\bar{z}}, \phi\right]_{k \ell}=2 \pi i \sum_{\alpha=1}^{N} S_{k \ell}^{\alpha} \delta^{(2)}\left(z-z_{\alpha}\right),
$$

where the $\mathrm{GL}(K, \mathbb{C})$ spin variables are defined as

$$
S_{k \ell}^{\alpha}=Q_{k}^{\alpha} \tilde{Q}_{\ell}^{\alpha}-\frac{m}{N} \delta_{k \ell}
$$

They are subject to the constraints

$$
\frac{1}{K} \sum_{k=1}^{K} Q_{k}^{\alpha} \tilde{Q}_{k}^{\alpha}=m_{\alpha}, \quad \sum_{\alpha=1}^{N} Q_{k}^{\alpha} \tilde{Q}_{k}^{\alpha}=m .
$$

The first constraint implies that the spins lie in a particular conjugacy class of $\mathrm{GL}(K, \mathbb{C})$ and the second constraint ensures that the diagonal components of $\phi_{k \ell}$ have vanishing residues. This defines the hyper-Kähler quotient description of the instanton moduli space $\mathcal{M}_{K, N}$.

Let us proceed to solve the Hitchin system defined on the dual torus. With a suitable choice of gauge, one can diagonalize the connection as

$$
A_{\bar{z}}=\frac{\pi i}{2\left(\bar{\omega}_{2} \omega_{1}-\bar{\omega}_{1} \omega_{2}\right)} \operatorname{diag}\left(x_{1}, x_{2}, \ldots, x_{K}\right),
$$

\footnotetext{
${ }^{3}$ The quantum mechanics on the moduli space of instantons on $\mathbb{R}^{2} \times T^{2}$ has recently been studied in [48].
} 
where $\omega_{i}$ are the half-periods of the dual torus and $x_{k}$ transform under a large gauge transformation into

$$
x_{k} \simeq x_{k}+2 n \omega_{1}+2 m \omega_{2}, \quad n, m \in \mathbb{Z} .
$$

One can then decompose the Higgs field $\phi$ into diagonal and off-diagonal pieces and look for doubly-periodic solutions with simple poles at the impurities. The answer is given in terms of a combination of Weierstraß functions as $[49,50]$

$$
\phi_{k \ell}(z)=\delta_{k \ell}\left[p_{k}+\sum_{\alpha=1}^{N} S_{k k}^{\alpha} \zeta\left(z-z_{\alpha}\right)\right]+\left(1-\delta_{k \ell}\right) \sum_{\alpha=1}^{N} S_{k \ell}^{\alpha} \frac{\sigma\left(x_{k \ell}+z-z_{\alpha}\right)}{\sigma\left(x_{k \ell}\right) \sigma\left(z-z_{\alpha}\right)} e^{x_{k \ell}\left(\psi(z)-\psi\left(z_{\alpha}\right)\right)}
$$

where $p_{k}$ are constants and we abbreviate $x_{k}-x_{\ell}$ as $x_{k \ell}$. The function $\psi(z)$ is defined as

$$
\psi(z)=\frac{1}{\bar{\omega}_{2} \omega_{1}-\bar{\omega}_{1} \omega_{2}}\left[\zeta\left(\omega_{2}\right)\left(\bar{\omega}_{1} z-\omega_{1} \bar{z}\right)-\zeta\left(\omega_{1}\right)\left(\bar{\omega}_{2} z-\omega_{2} \bar{z}\right)\right]
$$

One may readily check that this solution satisfies the required properties by recalling that the Weierstraß functions are quasi-periodic functions that transform as

$$
\zeta\left(z+2 \omega_{i}\right)=\zeta(z)+2 \zeta\left(\omega_{i}\right), \quad \sigma\left(z+2 \omega_{i}\right)=-\sigma(z) e^{2\left(z+\omega_{i}\right) \zeta\left(\omega_{i}\right)},
$$

and that $\zeta(z)$ has a simple pole at the origin while $\sigma(z)$ is regular there.

The canonical symplectic form (2.3) on the ambient space descends to the quotient space

$$
\omega=\sum_{k=1}^{K} d x_{k} \wedge d p_{k}+\sum_{k=1}^{K} \sum_{\alpha=1}^{N} d Q_{k}^{\alpha} \wedge d \tilde{Q}_{k}^{\alpha}
$$

The symplectic form defines the Poisson bracket among the variables $x_{k}, p_{k}, Q_{k}^{\alpha}, \tilde{Q}_{k}^{\beta}$

$$
\left\{x_{k}, p_{\ell}\right\}=\delta_{k \ell}, \quad\left\{Q_{k}^{\alpha}, \tilde{Q}_{\ell}^{\beta}\right\}=\delta^{\alpha \beta} \delta_{k \ell} .
$$

The hyper-Kähler quotient implies that gauge-invariant quantities that trivially Poissoncommute in the ambient space will automatically commute in the quotient space. The trace of powers of $\phi$ will form an infinite tower of Poisson-commuting Hamiltonians

$$
H_{n}=\frac{1}{n} \operatorname{tr} \phi^{n} .
$$

The Hamiltonians are encoded in the spectral curve, which is defined as the characteristic equation for $\phi$

$$
\Gamma(z, v) \equiv \operatorname{det}(v \mathbb{I}-\phi(z))=0 .
$$

The meromorphic differential $v d z$ is the Seiberg-Witten differential. The generators $\phi$ of the commuting conserved charges are known as the Lax matrix in the integrable systems literature. The quotient construction gives the resulting manifold the structure of an algebraic integrable system [49]. 


\subsection{The inhomogeneous spin Calogero-Moser model and its degenerate limits}

The classical integrable system describes $K$ non-relativistic particles on a doubly-periodic lattice which interact with pairwise elliptic potentials and spin exchanges. The Hamiltonian which contains the momentum quadratically as the non-relativistic kinetic term is given explicitly as $[49,50]$

$$
\begin{aligned}
H= & \sum_{k=1}^{K} p_{k}^{2}+\sum_{\ell \neq k}^{K} \sum_{\alpha=1}^{N} S_{k \ell}^{\alpha} S_{\ell k}^{\alpha} \wp\left(x_{k \ell}\right)+\sum_{k=1}^{K} \sum_{\beta \neq \alpha}^{N} S_{k k}^{\alpha} S_{k k}^{\beta}\left(\wp\left(z_{\alpha \beta}\right)-\zeta\left(z_{\alpha \beta}\right)^{2}\right) \\
& +\sum_{\ell \neq k}^{K} \sum_{\alpha \neq \beta}^{N} S_{k \ell}^{\alpha} S_{\ell k}^{\beta} \frac{\sigma\left(x_{k \ell}+z-z_{\alpha}\right)}{\sigma\left(x_{k \ell}\right) \sigma\left(z-z_{\alpha}\right)} e^{x_{k \ell} z_{\alpha \beta}}\left[\zeta\left(x_{k \ell}+z_{\alpha \beta}\right)-\zeta\left(x_{k \ell}\right)\right] .
\end{aligned}
$$

The variables $x_{k}$ and $p_{k}$ correspond to the position and momentum of the Calogero-Moser particles. The $\mathrm{GL}(K, \mathbb{C})$ spin variables can be traded with the $\mathrm{SL}(N, \mathbb{C})$ spin variables $S_{k}^{\alpha \beta}$ labeling the internal spin carried by each particle

$$
S_{k}^{\alpha \beta}=Q_{k}^{\alpha} \tilde{Q}_{k}^{\beta}-\frac{\delta^{\alpha \beta}}{N} \sum_{\gamma=1}^{N} Q_{k}^{\gamma} \tilde{Q}_{k}^{\gamma} .
$$

We will call the corresponding integrable model the inhomogeneous spin Calogero-Moser model. The two special gauge theories considered above correspond to special limits of this general model. In the following we will explore these models in detail.

There is an important subtlety that we need to address when quantizing the models. The integrable models that arise from gauge theory are complex integrable systems where the dynamical variables $x_{k}, p_{k}$ are complex-valued and the spin variables $S_{k}^{\alpha \beta}$ take value in representations of the complex Lie algebra $\mathfrak{s l}(N, \mathbb{C})$. One can impose different reality conditions to obtain inequivalent real integrable systems. This chooses a middle-dimensional subspace of the complex integrable system. Upon quantization these lead to quantum integrable systems with very different wave functions and spectra. For example, the spins can be chosen to lie in a compact $\mathfrak{s u}(N, \mathbb{R})$ representation or in a non-compact $\mathfrak{s l}(N, \mathbb{R})$ representation. One can also choose the particles to lie on the real axis or on the imaginary axis. In the asymptotic limit, we obtain the hyperbolic Sutherland or the trigonometric Sutherland model, respectively. The former has a continuous spectrum whereas the latter has a discrete spectrum. Because we wish to solve for the two-body S-matrices, we will choose the particles to lie on the real axis. We can then make a choice for the representation of the spin variables. As we will discuss in section 4 , this corresponds to conditions on the Seiberg-Witten curve where periods around various cycles become quantized.

The spin Calogero-Moser model. The model simplifies when we take all the inhomogeneities to coincide at the origin. In the brane picture, this corresponds to taking all the $N$ NS5-branes to be coincident. We reduce to the spin Calogero-Moser system [51-54]. The Hamiltonian is given by

$$
H=\sum_{k=1}^{K} \frac{p_{k}^{2}}{2}+\sum_{\ell>k}^{K} \sum_{\alpha, \beta=1}^{N} S_{k \ell}^{\alpha} S_{\ell k}^{\beta} \wp\left(x_{k}-x_{\ell}\right) .
$$


The Hubbard-Toda model. The Calogero-Moser particles interact with a pairwise potential. There is a special limit where only the nearest neighbors interact while others are suppressed exponentially. This is obtained by taking the particles to be far apart while tuning the coupling such that

$$
x_{k} \rightarrow X_{k}+k \log \mu^{2}, \quad \Lambda^{K}=\mu^{K} e^{2 \pi i \tau} \text { fixed. }
$$

This is known as the Inozemtsev limit [37]. In this limit, the Calogero-Moser system degenerates into the Toda chain where the particles interact via the nearest-neighbor exponential potential. The Inozemtsev limit of the inhomogeneous spin Calogero-Moser system gives rise to a new integrable system. The new system is a hybrid between the Toda chain and the Hubbard model. The Lax matrix is given by (see appendix A for details)

$$
L_{k \ell}(z)=L_{k \ell}^{\mathrm{Toda}}(z)+A_{k \ell}\left[\delta_{k \ell} \frac{t+1}{2(t-1)}+\Theta_{k \ell} \frac{t}{t-1}+\Theta_{\ell k} \frac{1}{t-1}\right], \quad t=e^{z} .
$$

Here $L^{\text {Toda }}$ is the Lax matrix for the Toda chain (A.10), the hopping operators $A_{k \ell}(1.6)$ satisfy

$$
\left\{A_{k \ell}, A_{m n}\right\}=\delta_{k n} A_{m \ell}-\delta_{m \ell} A_{k n},
$$

and $\Theta_{k \ell}$ is the discrete Heaviside function taking value 1 for $k>\ell$ and zero otherwise. The quadratic Hamiltonian can be written as

$$
\begin{aligned}
H_{2}= & H^{\text {Toda }}+\sum_{k=1}^{K} \frac{A_{k k}^{2}}{8}+\frac{t}{2(t-1)^{2}} \operatorname{tr} A^{2}+\frac{t+1}{2(t-1)} \sum_{k=1}^{K} p_{k} A_{k k} \\
& +\sum_{k=1}^{K-1} e^{\frac{X_{k}-X_{k+1}}{2}}\left(\frac{t}{t-1} A_{(k+1) k}-\frac{1}{t-1} A_{k(k+1)}\right)+\Lambda^{K} e^{\frac{X_{K}-X_{1}}{2}}\left(\frac{t}{t-1} A_{1 K}-\frac{1}{t-1} A_{K 1}\right) .
\end{aligned}
$$

Here $H^{\text {Toda }}$ is the Toda Hamiltonian (1.5). We define the Hubbard-Toda Hamiltonian such that the momentum only appears quadratically in the non-relativistic kinetic term. If we write $\mathrm{H}_{2}$ as

$$
H_{2}=\frac{\mathcal{H}_{2} t^{2}+\mathcal{H}_{1} t+\mathcal{H}_{0}}{(t-1)^{2}}
$$

then the coupled $p_{k} A_{k k}$ term can be eliminated by taking the linear combination $H^{\mathrm{HT}}=$ $\left(\mathcal{H}_{2}+\mathcal{H}_{0}\right) / 2$ :

$$
H^{\mathrm{HT}}=H^{\mathrm{Toda}}+\sum_{k=1}^{K} \frac{A_{k k}^{2}}{4}+\frac{1}{2}\left[\sum_{k=1}^{K-1} e^{\frac{x_{k}-X_{k+1}}{2}}\left(A_{(k+1) k}+A_{k(k+1)}\right)+\Lambda^{K} e^{\frac{x_{K}-X_{1}}{2}}\left(A_{1 K}+A_{K 1}\right)\right] .
$$

When the particles are frozen to their equilibrium configuration, it is the Hubbard model describing a lattice with spins hopping between neighboring sites. Having obtained the Hamiltonian for (1) the spin Calogero-Sutherland model, and (2) the Hubbard-Toda model, our next goal is to diagonalize them. Traditionally, this can be achieved using the quantum inverse scattering method $[55,56]$. We will review this method and apply it to the integrable models in the next section. 


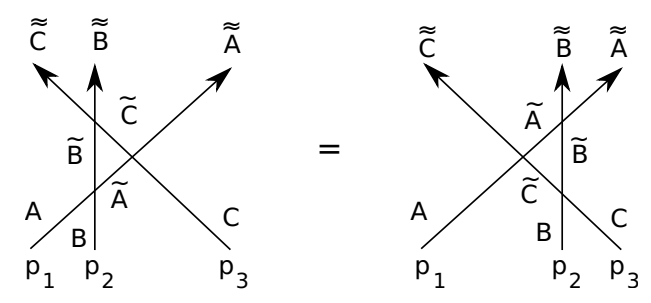

Figure 4. The Yang-Baxter relation.

\section{Solution by the quantum inverse scattering method}

The hallmark of quantum integrable models is factorized scattering, i.e., particle scatterings factorize into a series of two-body scatterings and individual momentum is conserved. As the models we consider are $\mathfrak{s l}(N)$-invariant systems, each particle transforms in an irreducible representation $h^{A}$ of $\mathfrak{s l}(N)$ labeled by an index $A$. Due to translational invariance, the two-body S-matrix $\mathbb{S}: h^{A} \otimes h^{B} \rightarrow h^{\tilde{A}} \otimes h^{\tilde{B}}$ only depends on the difference of the momenta and takes the form $\mathbb{S}_{A B}^{\tilde{A} \tilde{B}}\left(p_{k}-p_{\ell}\right)$. Consistency of scattering implies that the outcome is independent of the order in which the particles are scattered. This is shown in figure 4, from which one may read off the Yang-Baxter equation

$$
\left.\mathbb{S}_{\tilde{B} \tilde{C}}^{\tilde{\tilde{C}}} \tilde{\tilde{C}}\left(p_{2}-p_{3}\right) \mathbb{S}_{\tilde{A} C}^{\tilde{\tilde{A}} \tilde{C}}\left(p_{1}-p_{3}\right) \mathbb{S}_{A B}^{\tilde{A} \tilde{B}}\left(p_{1}-p_{2}\right)=\mathbb{S}_{\tilde{A} \tilde{B}}^{\tilde{\tilde{A}}}\left(p_{1}-p_{2}\right) \mathbb{S}_{A \tilde{C}}^{\tilde{A} \tilde{\tilde{C}}}\left(p_{1}-p_{3}\right) \mathbb{S}_{B C}^{\tilde{B} C} p_{2}-p_{3}\right)
$$

The Yang-Baxter equation strongly constrains the form of the S-matrix. It completely fixes the S-matrix up to a scalar factor:

$$
\mathbb{S}_{A B}^{\tilde{A} \tilde{B}}(p)=S_{0}(p) \mathbb{R}_{A B}^{\tilde{A} \tilde{B}}(p) .
$$

For example, when the particles are in the fundamental representation of $\mathfrak{s u}(N)$ labeled by $\alpha=1, \ldots, N$, the only invariant tensors are the identity operator $\mathbb{I}_{\alpha \beta}^{\tilde{\alpha} \tilde{\beta}}=\delta_{\alpha}^{\tilde{\alpha}} \delta_{\beta}^{\tilde{\beta}}$ and the permutation operator $\mathbb{P}_{\alpha \beta}^{\tilde{\alpha} \tilde{\beta}}=\delta_{\alpha}^{\tilde{\beta}} \delta_{\beta}^{\tilde{\alpha}}$. The R-matrix can be constructed from a linear combination of the invariant tensors $\mathbb{R}(p)=a(p) \mathbb{I}+b(p) \mathbb{P}$. The relative coefficients can then be constrained by the Yang-Baxter equation, and we obtain the fundamental $\mathfrak{s u}(N)$ R-matrix

$$
\mathbb{R}(p)=\frac{p \mathbb{I}+i \mathbb{P}}{p+i} .
$$

The scalar factor $S_{0}(p)$ does not depend on the Yang-Baxter equation and is more tricky to determine. It is usually fixed by imposing unitarity and crossing symmetry. Even then, there can be multiple solutions to the crossing equation and one needs to further impose conditions on the bound-state spectrum, e.g., the assumption of absence of poles and zeros in the physical momentum region. For particle models where the explicit Hamiltonians are known, we can in principle determine the full S-matrix by directly solving the Schrödinger equations. The components of the S-matrix can then be read off from the asymptotics of the wave functions. In practice, however, diagonalizing the matrix Schrödinger equations can be difficult. We will illustrate the use of both methods for the spin Calogero-Moser model and the Hubbard-Toda model in this section. 
We begin by solving the problem on an infinite line. The asymptotic states are welldefined in the infinite-volume limit. Assuming that the particles are well-separated, the $K$-particle wave function can be written as

$$
\psi_{A_{1} \cdots A_{K}}\left(x_{1}, \ldots, x_{K}\right) \simeq \sum_{\sigma \in \operatorname{Sym}(K)} \mathbb{S}_{\sigma} \exp \left(i \sum_{k=1}^{K} p_{\sigma(k)} x_{k}\right)
$$

Here $\mathbb{S}_{\sigma}$ denotes that if two permutations $\sigma$ and $\sigma^{\prime}$ differs by swapping $k$ and $\ell$, then $\mathbb{S}_{\sigma} / \mathbb{S}_{\sigma^{\prime}}=\mathbb{S}_{k \ell}\left(p_{k}-p_{\ell}\right)$. We then place the system on a large circle of radius $L \gg 1$ and impose a twisted periodic boundary condition

$$
\psi_{A_{1} \cdots A_{K}}\left(x_{2}, \ldots, x_{K}, x_{1}+L\right)=\mathbb{T}_{A_{1} B_{1}}^{-1} \psi_{B_{1} A_{2} \cdots A_{K}}\left(x_{1}, \ldots, x_{K}\right),
$$

where $\mathbb{T}=\exp \left(2 \pi i \tau S^{z}\right)$ is a twisted periodic boundary condition that rotates the spin at the end of the chain in the preferred $z$-direction. For $L \gg 1$, we have

$$
\psi_{A_{1} \cdots A_{K}}\left(x_{2}, \ldots, x_{K}, x_{1}+L\right) \simeq e^{-i p_{1} L} \mathbb{S}_{12}\left(p_{1}-p_{2}\right) \cdots \mathbb{S}_{1 K}\left(p_{1}-p_{K}\right) \psi_{A_{1} \cdots A_{K}}\left(x_{1}, \ldots, x_{K}\right) .
$$

The asymptotic Bethe ansatz dictates that the phase shift acquired by a particle around the system is equal to the product of S-matrices with all the other particles.

$$
e^{i p_{k} L}|\psi\rangle=\mathbb{T} \prod_{\ell \neq k}^{K} \mathbb{S}_{k \ell}\left(p_{k}-p_{\ell}\right)|\psi\rangle .
$$

The solution in terms of the asymptotic Bethe ansatz is valid for large system size $L \gg 1$ up to sub-leading finite-size effects of order $\exp \left(-8 \pi^{2} / g^{2}\right)$. This is also the starting point of the finite-volume problem, where we go to the mirror model and formulate a set of thermodynamic Bethe ansatz equations. As we shall explain below, this is closely related to the diagonalization problem of the integrable spin chain.

\subsection{The spin Calogero-Moser model}

Let us solve the spin Calogero-Moser model (2.18) using the asymptotic Bethe ansatz. This model is quantum integrable [57]. In the large-volume limit, one period in the lattice grows to infinity. The Hamiltonian becomes that of the spin Calogero-Sutherland model:

$$
H=\sum_{k=1}^{K} \frac{p_{k}^{2}}{2}+\sum_{\ell>k}^{K} \sum_{\alpha, \beta=1}^{N} \frac{S_{k \ell}^{\alpha} S_{\ell k}^{\beta}}{4 \sinh ^{2} \frac{x_{k \ell}}{2}}
$$

The particles now move on the real line and interact with a hyperbolic potential. The Hamiltonian can be rewritten in terms of the $\mathfrak{s l}(N)$ spin variables using (2.5) and (2.17) as

$$
\sum_{\alpha, \beta=1}^{N} S_{k \ell}^{\alpha} S_{\ell k}^{\beta}=\sum_{\alpha, \beta=1}^{N} S_{k}^{\alpha \beta} S_{\ell}^{\beta \alpha}+\frac{m^{2}}{N}
$$


Note that this coincides with the square of the total spin when $N=2$ :

$$
\left(S_{k}+S_{\ell}\right)^{2}=\sum_{\alpha, \beta=1}^{N} S_{k}^{\alpha \beta} S_{\ell}^{\beta \alpha}+m^{2}-\frac{m^{2}}{N} .
$$

To quantize the model, we promote the spin variables $S_{k}$ to the spin operators $\hat{S}_{k}$. For simplicity, we take all sites to be in the same spin- $s$ representation of $\mathfrak{s u}(2)$. Integrability implies that the particle interactions of the model can be factorized into a series of twobody scatterings. It is useful to go to the center-of-mass frame such that $p_{1}=-p_{2}=p / 2$ and $x=x_{2}-x_{1}$. The S-matrix can be determined by solving the matrix Schrödinger equation

$$
\left(-\frac{d^{2}}{d x^{2}} \mathbb{I}+\mathbb{V}(x)\right) \vec{\psi}(x)=E \vec{\psi}(x)
$$

where the potential is written in terms of the total spin operator as

$$
\mathbb{V}(x)=\frac{\left(\hat{S}_{1}+\hat{S}_{2}\right)^{2}}{4 \sinh ^{2}\left(\frac{x}{2}\right)} .
$$

In each irreducible subspace of the total spin operator labeled by the eigenvalue $s$, we obtain a scalar Schrödinger problem with a hyperbolic potential and coupling $s(s+1)$. The scattering phase of particles in a hyperbolic potential is known explicitly [58]

$$
\mathbb{S}(p)=\frac{\Gamma(1+\hat{S}-i p) \Gamma(1+i p)}{\Gamma(1+\hat{S}+i p) \Gamma(1-i p)} \mathbb{P} .
$$

The key observation is that the Calogero-Sutherland particle S-matrix (3.13) coincides with the universal R-matrix of the Heisenberg $\mathfrak{s u}(2)$ spin chain [59]. In this case the scalar part of the S-matrix is

$$
S_{0}(p)=\frac{\Gamma(1+2 s-i p) \Gamma(1+i p)}{\Gamma(1+2 s+i p) \Gamma(1-i p)}
$$

such that $\mathbb{R}(p)$ acting on the ground state with all spins up is the identity operator. We can then proceed to solve the model for a set of Bethe ansatz equations using the quantum inverse scattering method.

Consider a ghost particle labeled by 0 with momentum $p$ scattering with the other $K$ particles. We define the fundamental monodromy matrix $\hat{T}_{0}(p)$ as the product of Rmatrices and the transfer matrix $\hat{t}(p)=\operatorname{tr}_{0} \hat{T}_{0}(p)$ as its trace over the ghost particle:

$$
\hat{T}_{0}(p)=\mathbb{T} \prod_{k=1}^{K} \mathbb{R}_{0 k}\left(p-p_{k}\right) .
$$

Integrability of the model follows because the fundamental monodromy matrix likewise satisfies the Yang-Baxter equation. This then implies that the fundamental transfer matrices commute at different values of the spectral parameters $\left[\hat{t}(p), \hat{t}\left(p^{\prime}\right)\right]=0$, and generate the quantum commuting Hamiltonians. 

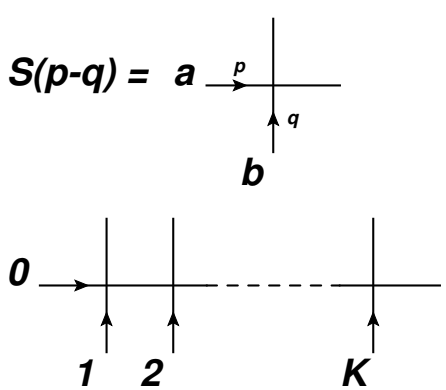
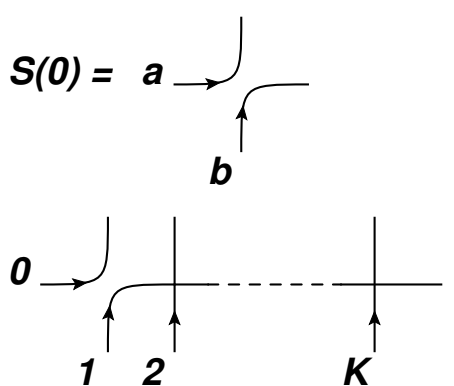

Figure 5. The S-matrix becomes the permutation operator when the two particles have the same momentum. The transfer matrix describes the scattering of a ghost particle with the physical particles. When the ghost particle has the same momentum as a physical particle, they can be swapped and the fundamental transfer matrix becomes the scattering of that physical particle with the other physical particles.

If we evaluate the fundamental monodromy matrix at one of the particle momenta, then because $\mathbb{S}_{0 \ell}(0)=\mathbb{P}_{0 \ell}$, the ghost particle swaps with that physical particle. For $p=p_{1}$, we may commute $\mathbb{P}_{01}$ to the right using $\mathbb{P}_{0 \ell} \mathbb{P}_{0 k} \mathbb{P}_{0 \ell}=\mathbb{P}_{k \ell}$ to obtain

$$
\prod_{k=1}^{K} \mathbb{S}_{0 k}\left(p_{1}-p_{k}\right)=\mathbb{S}_{12}\left(p_{1}-p_{2}\right) \mathbb{S}_{13}\left(p_{1}-p_{3}\right) \cdots \mathbb{S}_{1 K}\left(p_{1}-p_{K}\right) \mathbb{P}_{01}
$$

and similarly for arbitrary $p=p_{k}$. We recover the products of S-matrices in (3.7) by tracing out the ghost particle since $\operatorname{tr}_{0} \mathbb{P}_{01}=1$. Graphically, this can be represented as in figure 5 .

Thus diagonalizing the product of S-matrices is equivalent to diagonalizing the fundamental transfer matrix. The latter problem can be solved using the nested algebraic Bethe ansatz $[60,61]$. In this approach, we first introduce an auxiliary $\mathfrak{s u}(2)$ space for the ghost particle. We define the auxiliary monodromy matrix $\hat{T}_{a}$ as in (3.15), where now the ghost particle lives in the auxiliary space. When the physical particles are in the spin- $1 / 2$ representation, the fundamental monodromy matrix coincides with the auxiliary monodromy matrix. The fundamental and the auxiliary monodromy matrices satisfy the Yang-Baxter equation

$$
\mathbb{R}_{a 0}(p-q) \hat{T}_{a}(p) \hat{T}_{0}(q)=\hat{T}_{0}(q) \hat{T}_{a}(p) \mathbb{R}_{a 0}(p-q),
$$

implying that $\left[\hat{t}(p), \hat{t}_{a}\left(p^{\prime}\right)\right]=0$ thus we can simultaneously diagonalize $\hat{t}$ and $\hat{t}_{a}$. It turns out to be simpler to first find eigenstates of the auxiliary transfer matrix and then calculate the eigenvalue of the fundamental transfer matrix on these eigenstates. We write the auxiliary monodromy matrix as a $2 \times 2$ matrix over the auxiliary space where each entry is an operator acting on the physical space

$$
\hat{T}_{a}(p)=\left(\begin{array}{cc}
\hat{A}(p) & \hat{B}(p) \\
\hat{C}(p) & e^{2 \pi i \tau} \hat{D}(p)
\end{array}\right)
$$


such that the transfer matrix is simply $\hat{t}_{a}=\hat{A}+e^{2 \pi i \tau} \hat{D}$. The entries of the auxiliary monodromy matrix can be found using the explicit expressions of the R-matrix

$$
\begin{aligned}
\mathbb{R}_{0 k}(p) & =\frac{1}{p+i}\left(p \mathbb{I}+i \vec{\sigma} \cdot \vec{S}_{k}\right) \\
& =\frac{1}{p+i}\left(\begin{array}{cc}
p+i S_{k}^{z} & i S_{k}^{-} \\
i S_{k}^{+} & p-i S_{k}^{z}
\end{array}\right) .
\end{aligned}
$$

Thus we define the nested pseudo-vacuum $\Omega\left(\left\{p_{k}\right\}\right)$ as the highest-weight state annihilated by $\hat{C}(p)$. It is also an eigenstate of the transfer matrix

$$
\hat{A}(p) \Omega=\prod_{k=1}^{K} \frac{p-p_{k}+i s}{p-p_{k}+i} \Omega, \quad \hat{D}(p) \Omega=\prod_{k=1}^{K} \frac{p-p_{k}-i s}{p-p_{k}+i} \Omega .
$$

The eigenstates of $\hat{t}_{a}$ are generated by acting on the nested pseudo-vacuum with the creation operators labeled by the magnon rapidities $\lambda_{i}$ :

$$
\Phi\left(\left\{\lambda_{i}\right\},\left\{p_{k}\right\}\right)=\hat{B}\left(\lambda_{1}\right) \cdots \hat{B}\left(\lambda_{M}\right) \Omega\left(\left\{p_{k}\right\}\right)
$$

The Yang-Baxter equation (3.1) implies that the commutation relations are

$$
\begin{aligned}
& \hat{A}(p) \hat{B}(\lambda)=\frac{p-\lambda-i}{p-\lambda} \hat{B}(\lambda) \hat{A}(p)+\frac{i}{p-\lambda} \hat{B}(p) \hat{A}(\lambda) \\
& \hat{D}(p) \hat{B}(u)=\frac{p-\lambda+i}{p-\lambda} \hat{B}(\lambda) \hat{D}(p)-\frac{i}{p-\lambda} \hat{B}(p) \hat{D}(\lambda) .
\end{aligned}
$$

By commuting $\hat{A}$ and $\hat{D}$ past the creation operators using the commutation relations, we find that the eigenvalue of the auxiliary transfer matrix is

$$
t_{a}(p)=\prod_{k=1}^{K} \frac{p-p_{k}+i s}{p-p_{k}+i} \prod_{i=1}^{M} \frac{p-\lambda_{i}-i}{p-\lambda_{i}}+e^{2 \pi i \tau} \prod_{k=1}^{K} \frac{p-p_{k}-i s}{p-p_{k}+i} \prod_{i=1}^{M} \frac{p-\lambda_{i}+i}{p-\lambda_{i}}
$$

The eigenvalue of the fundamental transfer matrix on the Bethe state $\Phi\left(\left\{\lambda_{i}\right\},\left\{p_{k}\right\}\right)$ is known to be [59]

$$
t(p)=\prod_{i=1}^{M} \frac{p-\lambda_{i}-i s}{p-\lambda_{i}+i s}+\mathcal{O}\left(p^{K}\right)
$$

We arrive at the asymptotic Bethe ansatz equation from (3.7)

$$
e^{i p_{k} L}=\prod_{\ell=1}^{K} \frac{\Gamma\left(1+2 s-i p_{k \ell}\right) \Gamma\left(1+i p_{k \ell}\right)}{\Gamma\left(1+2 s+i p_{k \ell}\right) \Gamma\left(1-i p_{k \ell}\right)} \prod_{i=1}^{M} \frac{p_{k}-\lambda_{i}-i s}{p_{k}-\lambda_{i}+i s}
$$

We see that the eigenvalue of the S-matrix factorizes into two parts: one that represents the scattering of the particles and one that represents the scattering of the particles with 
the magnons. There is another set of Bethe ansatz equations for the magnons. This follows from requiring the auxiliary transfer function (3.23) to have vanishing residue at $p=\lambda_{i}$.

$$
\prod_{k=1}^{K} \frac{\lambda_{i}-p_{k}+i s}{\lambda_{i}-p_{k}-i s}=e^{2 \pi i \tau} \prod_{j \neq i}^{M} \frac{\lambda_{i}-\lambda_{j}+i}{\lambda_{i}-\lambda_{j}-i} .
$$

This is the Bethe ansatz equation describing the scattering of magnons on a spin chain with inhomogeneity $p_{k}$ at each site.

\subsection{The Hubbard-Toda model}

The Hubbard-Toda model is a dynamical lattice of Toda particles with spins that can hop between neighboring sites. This new feature also makes diagonalizing the problem difficult. The model is classically integrable as it arises from the Inozemtsev limit of the inhomogeneous spin Calogero-Moser system. We explicitly verify the classical integrability of this model in appendix B. While we do not yet have a proof of quantum integrability, we will assume that the model is also integrable at the quantum level and solve for the two-body S-matrix.

\subsubsection{The non-compact $\mathfrak{s l}(2)$ model}

We begin by focusing on the non-compact model where each particle carries canonicallycommuting bosonic oscillators $Q_{k}^{\alpha}=\left(a_{k}, b_{k}^{\dagger}\right), \tilde{Q}_{k}^{\alpha}=\left(a_{k}^{\dagger},-b_{k}\right)$ such that

$$
\left[Q_{k}^{\alpha}, \tilde{Q}_{\ell}^{\beta}\right]=\delta_{k \ell} \delta^{\alpha \beta} \Longleftrightarrow\left[a_{k}, a_{k}^{\dagger}\right]=\left[b_{k}, b_{k}^{\dagger}\right]=1 .
$$

The $\mathfrak{s l}(2, \mathbb{R}) \simeq \mathfrak{s u}(1,1)$ algebra is generated by the spin variables $S_{k}^{\alpha \beta}(2.17)$. In matrix form,

$$
S_{k}^{\alpha \beta}=\left(\begin{array}{cc}
\frac{1}{2}\left(N_{k}^{a}+N_{k}^{b}+1\right) & -a_{k} b_{k} \\
b_{k}^{\dagger} a_{k}^{\dagger} & -\frac{1}{2}\left(N_{k}^{a}+N_{k}^{b}+1\right)
\end{array}\right),
$$

where $N_{k}^{a}=a_{k}^{\dagger} a_{k}$ and $N_{k}^{b}=b_{k}^{\dagger} b_{k}$ are the number operators. The quadratic Casimir is $S_{k}^{2}=s_{k}\left(s_{k}+1\right)$, where $s_{k}=\left(N_{k}^{b}-N_{k}^{a}-1\right) / 2$.

For two sites, we take the total spin operator $S_{G}=S_{1}+S_{2}$ as the global $\mathfrak{s u}(1,1)_{G}$ generator. The quadratic Casimir $S_{G}^{2}=s_{G}\left(s_{G}+1\right)$ is determined by the tensor product

$$
V_{s_{1}} \otimes V_{s_{2}}=\bigoplus_{s_{G}=s_{1}+s_{2}+1}^{\infty} V_{s_{G}} .
$$

The two-body Hamiltonian is (2.24)

$$
H^{\mathrm{HT}}=\left(\frac{p}{2}\right)^{2}+\frac{1}{4}\left(A_{11}^{2}+A_{22}^{2}\right)+\frac{1}{2}\left(A_{12}+A_{21}\right) e^{-x / 2}+e^{-x} .
$$

Our goal is to diagonalize the hopping term $A_{12}+A_{21}$. We write it as the sum of two terms $T_{+}=A_{12}$ that moves a type- $a$ spin from the first site to the second site and moves a type- $b$ spin from the second site to the first site, and $T_{-}=A_{21}$, which does the opposite. 


\begin{tabular}{|ccc|}
\hline $\mathfrak{s u}(1,1)_{G}$ & $\mathfrak{s u}(2)_{A}$ & Lowest-weight states \\
\hline 0 & 0 & $(0,0)$ \\
1 & 1 & $(B, A)$ \\
2 & 2 & $\left(B^{2}, A^{2}\right)$ \\
$\vdots$ & $\vdots$ & $\vdots$ \\
\hline
\end{tabular}

Table 2. Bases of two-particle states in the $\mathfrak{s u}(1,1)$ Hubbard-Toda model. For ease of notation, we use $\left(A^{N_{1}^{a}} B^{N_{1}^{b}}, A^{N_{2}^{a}} B^{N_{2}^{b}}\right)$ to denote a state with $N_{k}^{a}$ type- $a$ spins and $N_{k}^{b}$ type- $b$ spins at site $k$. The ladder operators $T_{ \pm}$hop spins between the two sites.

First, observe that if we define the difference between spins at two sites as $T_{z}=s_{1}-s_{2}$, then $T_{+}$increases $T_{z}$ by one unit and $T_{-}$decreases $T_{z}$ by one unit. Hence $T_{ \pm}$and $T_{z}$ are generators of an auxiliary $\mathfrak{s u}(2)$ symmetry algebra

$$
\left[T_{z}, T_{ \pm}\right]= \pm T_{ \pm}, \quad\left[T_{+}, T_{-}\right]=2 T_{z} .
$$

The total spin $\bar{s}=s_{1}+s_{2}+1$ is conserved under the action by $T_{ \pm}$so commutes with them. The Hamiltonian (3.30) can then be written in terms of the auxiliary $\mathfrak{s u}(2)$ generators as

$$
H^{\mathrm{HT}}=\left(\frac{p}{2}\right)^{2}+\frac{1}{4}\left(s_{A}^{2}-T_{z}^{2}\right)+T_{x} e^{-x / 2}+e^{-x} .
$$

The auxiliary $\mathfrak{s u}(2)$ symmetry generators commutes with the global $\mathfrak{s u}(1,1)$ symmetry generators. Remarkably, they have the same quadratic Casimirs and are labeled by the same $s$. This implies that the tensor product of two states can be decomposed as

$$
\bigoplus_{s_{1}=-\frac{1}{2}}^{\infty} V_{s_{1}} \otimes \bigoplus_{s_{2}=-\frac{1}{2}}^{\infty} V_{s_{2}}=\bigoplus_{s=0}^{\infty} V_{s} \otimes W_{s},
$$

where $W_{s}$ is a spin- $s$ representation of the auxiliary $\mathfrak{s u}(2)$ symmetry that counts the degeneracies of the spin- $s$ representation of the global $\mathfrak{s u}(1,1)$ symmetry. For concreteness, we present the multiplet structure in table 2 .

The S-matrix acting on the tensor product also decomposes into

$$
\mathbb{S}(p)=\sum_{s=\bar{s}}^{\infty} \mathcal{P}_{s} \mathbb{S}_{s}(p),
$$

where $\mathcal{P}_{s}$ projects onto the irreducible subspace $V_{s} . \mathbb{S}_{s}(p)$ is the operator acting on $W_{s} \otimes$ $C^{\infty}(\mathbb{R})$, naturally given by the $(2 s+1)$-component wave function $\vec{\psi}=\left(\psi_{s}, \ldots, \psi_{-s}\right)$ and obeying the matrix Schrödinger equation (3.11) with the Hubbard-Toda potential (3.32).

Note that the linear combination $H_{1}=-\mathcal{H}_{2}+\mathcal{H}_{0}$ from (2.23) defines another commuting Hamiltonian

$$
H_{1}=\frac{1}{2} T_{z} p-T_{y} e^{-x / 2}
$$

that asymptotes to $T_{z} p / 2$ as $x \rightarrow \infty$. Because of the existence of the conserved charge $H_{1}$, $\Delta s=T_{z}$ is preserved in scattering. The S-matrix does not mix the various components 
of the wave function labeled by $\Delta s$ and can be written in a block diagonal form with $S(p ; \Delta s, s)$ on the diagonals

$$
\mathbb{S}(p)=\operatorname{diag}(S(p ; s, s), S(p ; s-1, s), \ldots, S(p ;-s, s)) .
$$

The form of the S-matrix can be fixed assuming integrability. The Yang-Baxter relation (3.1) fixes the form of $\mathbb{S}_{s}(p)$ up to an overall scalar factor

$$
\mathbb{S}(p)=S_{0}(p) \sum_{s=\bar{s}}^{\infty} \mathcal{P}_{s} \mathbb{R}_{s}(p)
$$

Here $\mathbb{R}_{s}(p)$ is given by the universal $\mathfrak{s l}(2)$ R-matrix [59]

$$
\mathbb{R}_{s}(p)=(-1)^{s-\bar{s}} \frac{\Gamma(-s-i p) \Gamma(-\bar{s}+i p)}{\Gamma(-s+i p) \Gamma(-\bar{s}-i p)}
$$

where we have chosen the normalization such that $\mathbb{R}_{\bar{s}}=1$. The model can then be solved using the quantum inverse scattering method by diagonalizing the transfer matrix, as discussed in the last section, leading to the following set of nested Bethe ansatz equations

$$
\begin{aligned}
e^{i p_{k} L} & =\prod_{\ell \neq k}^{K} S_{0}\left(p_{k}-p_{\ell} ; s_{k}, s_{\ell}\right) \prod_{i=1}^{M} \frac{p_{k}-\lambda_{i}+i s_{k}}{p_{k}-\lambda_{i}-i s_{k}} \\
\prod_{k=1}^{K} \frac{\lambda_{i}-p_{k}+i s_{k}}{\lambda_{i}-p_{k}-i s_{k}} & =e^{2 \pi i \tau} \prod_{j \neq i}^{M} \frac{\lambda_{i}-\lambda_{j}+i}{\lambda_{i}-\lambda_{j}-i} .
\end{aligned}
$$

The Yang-Baxter equation alone, however, does not determine the scalar factor in the S-matrix. To fix the scalar factor, one needs to directly solve the matrix Schrödinger equation in the Hubbard-Toda potential, as we have done for the spin Calogero-Sutherland potential in the previous section. When we attempt to do so, we encounter a difficulty that the potential is not diagonal and the matrix becomes larger for higher spins. We will attempt to tackle the problem directly for the lower spins in the next section. As we shall see, an indirect method using the Bethe/Gauge correspondence gives a prediction for the full scalar factor for arbitrary spins. It is given by

$$
S_{0}\left(p ; s_{k}, s_{\ell}\right)=\frac{\Gamma(1+\Delta s+i p) \Gamma(1-\Delta s+i p) \Gamma(-\bar{s}-i p)}{\Gamma(1+\Delta s-i p) \Gamma(1-\Delta s-i p) \Gamma(-\bar{s}+i p)} .
$$

Note that the $\bar{s}$-dependent parts in the scalar factor exactly cancel with those in the universal R-matrix, such that the S-matrix is a function only of $\Delta s$ and $s$.

$$
\mathbb{S}(p ; \Delta s, s)=\frac{\Gamma(1+\Delta s+i p) \Gamma(1-\Delta s+i p)}{\Gamma(1+\Delta s-i p) \Gamma(1-\Delta s-i p)} \sum_{s=\bar{s}}^{\infty} \mathcal{P}_{s} \frac{\Gamma(-s-i p)}{\Gamma(-s+i p)}
$$

The result agrees with the direct method and provides a convincing evidence that the Bethe/Gauge correspondence is an effective tool for solving quantum integrable systems. 


\subsubsection{Solving the matrix Schrödinger equation}

Let us attempt to directly diagonalize

$$
H^{\mathrm{HT}}=\left(\frac{p}{2}\right)^{2}+\frac{1}{4}\left(s_{A}^{2}-T_{z}^{2}\right)+T_{x} e^{-x / 2}+e^{-x},
$$

by solving for the exact wave function and reading off the scattering phase $S\left(p ; \Delta s, s_{A}\right)$ from the asymptotics. The first difficulty we encounter is that $T_{z}$ and $T_{x}$ cannot be simultaneously diagonalized in general, and we have to solve a matrix Schrödinger equation. Asymptotically, $H^{\mathrm{HT}} \rightarrow(p / 2)^{2}+\left(s_{A}^{2}-T_{z}^{2}\right) / 4$ so it acts as a free Hamiltonian on the spin $\Delta s$ component of the wave function $\vec{\psi}$. This determines the dispersion relation:

$$
E=\left(\frac{p_{\Delta s}}{2}\right)^{2}+\frac{s_{A}^{2}-(\Delta s)^{2}}{4} .
$$

Spin 0. For spin 0, the problem reduces to a scalar Schrödinger equation with the Liouville potential

$$
-\psi^{\prime \prime}(x)+e^{-x} \psi(x)=\left(\frac{p}{2}\right)^{2} \psi(x) .
$$

The wave function is exactly solvable in terms of modified Bessel function of the second type. Up to an arbitrary normalization,

$$
\psi(x)=K_{i p}\left(2 e^{-\frac{x}{2}}\right) .
$$

It oscillates at $x \rightarrow \infty$ and exponentially decays at $x \rightarrow-\infty$ as is expected from the Liouville potential. The S-matrix can be read off from the ratio of the left-moving and the right-moving modes at $x \rightarrow \infty$

$$
S(p ; 0,0)=-\frac{\Gamma(1+i p)}{\Gamma(1-i p)} .
$$

Spin 1/2. For the spin- $1 / 2$ representation, $T_{x}, T_{y}, T_{z}$ are the Pauli matrices and we obtain a system of two coupled Schrödinger equations. We can decouple the equations in the basis $\psi_{ \pm}=\psi_{1 / 2} \pm \psi_{-1 / 2}$ in which $T_{x}$ is diagonal

$$
-\psi_{ \pm}^{\prime \prime}(x)+\left(e^{-x} \pm \frac{1}{2} e^{-\frac{x}{2}}\right) \psi_{ \pm}(x)=\left(\frac{p}{2}\right)^{2} \psi_{ \pm(x)} .
$$

It is an exactly solvable potential of Morse type and the wave functions are given in terms of confluent hypergeometric functions as

$$
\psi_{ \pm}(x)=e^{-2 e^{-\frac{x}{2}}-\frac{i p x}{2}} U\left(\frac{1}{2} \pm \frac{1}{2}+i p, 1+2 i p, 4 e^{-\frac{x}{2}}\right) .
$$

Looking at its asymptotics as $x \rightarrow \infty$, we can read off the S-matrix as before

$$
S\left(p ; \pm \frac{1}{2}, \frac{1}{2}\right)=\frac{\Gamma\left(\frac{1}{2}+i p\right)}{\Gamma\left(\frac{1}{2}-i p\right)},
$$

which agrees precisely with our prediction (3.41). Note that the S-matrix has a simple pole at $p=i / 2$, which is usually indicative of a bound state. However, $\psi_{-}$with this value of $p$ grows instead of decays at infinity. One can see why this is so because the state has energy $-1 / 16$, which touches the bottom of the potential well. It has no zero-point energy so cannot be a bound state. 
Spin 1. For the spin-1 representation, we have to solve the $3 \times 3$ coupled matrix Schrödinger equations

$$
\left(\begin{array}{ccc}
-d^{2}+e^{-x} & \frac{1}{\sqrt{2}} e^{-\frac{x}{2}} & \\
\frac{1}{\sqrt{2}} e^{-\frac{x}{2}} & -d^{2}+e^{-x}+\frac{1}{4} & \frac{1}{\sqrt{2}} e^{-\frac{x}{2}} \\
& \frac{1}{\sqrt{2}} e^{-\frac{x}{2}} & -d^{2}+e^{-x}
\end{array}\right)\left(\begin{array}{c}
\psi_{1} \\
\psi_{0} \\
\psi_{-1}
\end{array}\right)=E\left(\begin{array}{c}
\psi_{1} \\
\psi_{0} \\
\psi_{-1}
\end{array}\right)
$$

Note that if we define $\psi_{ \pm}=\left(\psi_{1} \pm \psi_{-1}\right) / \sqrt{2}$, then the equation for $\psi_{-}$decouples and the problem reduces to a $2 \times 2$ Hamiltonian acting on $\vec{\psi}=\left(\psi_{+}, \psi_{0}\right)$,

$$
H(x) \vec{\psi}(x)=E \vec{\psi}(x), \quad H(x)=\left(\begin{array}{cc}
-d^{2}+e^{-x} & e^{-\frac{x}{2}} \\
e^{-\frac{x}{2}} & -d^{2}+e^{-x}+\frac{1}{4}
\end{array}\right) .
$$

We perform a Darboux (supersymmetric) transform to decouple the equations, and recover the eigenvector $\vec{\psi}$ from the diagonal basis. This goes as follows [62, 63]. We look for a matrix $Q(x)=d+A(x)$ such that the new state $\vec{\phi}(x)=Q(x) \vec{\psi}(x)$ is an eigenstate of a diagonalized Hamiltonian $\widetilde{H}$ with energy $E$. In the language of supersymmetric quantum mechanics, $Q$ is the supersymmetric transform that intertwines the pair of supersymmetric Hamiltonians as $Q H=\widetilde{H} Q$. Solving for $Q$ and $\widetilde{H}$, we find

$$
Q(x)=\left(\begin{array}{cc}
d & e^{-\frac{x}{2}} \\
e^{-\frac{x}{2}} & d+\frac{1}{2}
\end{array}\right), \quad \widetilde{H}(x)=\left(\begin{array}{ll}
-d^{2}+e^{-x} & \\
& -d^{2}+e^{-x}+\frac{1}{4}
\end{array}\right) .
$$

The decoupled equations reduce to the spin-0 problem and can be easily solved as before. The original wave functions can be obtained from $\vec{\phi}$ by inverting the Darboux transform:

$$
\begin{aligned}
& \psi_{+}(x)=y\left[2 c_{0} K_{i p_{0}}(y)-c_{+}\left(K_{i p_{1}-1}(y)+K_{i p_{1}+1}(y)\right)\right], \quad y=2 e^{-\frac{x}{2}} \\
& \psi_{0}(x)=y\left[2 c_{+} K_{i p_{1}}(y)+c_{0}\left(e^{\frac{x}{2}} K_{i p_{0}}(y)-K_{i p_{0}-1}(y)-K_{i p_{0}+1}(y)\right)\right] .
\end{aligned}
$$

$\psi_{-}$can also be solved because it is decoupled from $\psi_{+}$and $\psi_{0}$. Up to a normalization constant,

$$
\psi_{-}(x)=c_{-} K_{i p_{1}}\left(2 e^{-\frac{x}{2}}\right) .
$$

The S-matrix can again be read off from the asymptotics of the wave functions. It agrees with our prediction when $c_{-}=0$

$$
S\left(p_{1} ; \pm 1,1\right)=\frac{\Gamma\left(1+i p_{1}\right)}{\Gamma\left(1-i p_{1}\right)}, \quad S\left(p_{0} ; 0,1\right)=-\frac{\left(1-i p_{0}\right) \Gamma\left(1+i p_{0}\right)}{\left(1+i p_{0}\right) \Gamma\left(1-i p_{0}\right)} .
$$

One may ask if there is any bound state corresponding to zeros or poles of the S-matrix. $S\left(p_{0} ; 0,1\right)$ has a double pole at $p_{0}=i(E=0)$ where

$$
\psi_{+}(x)=4 e^{-\frac{x}{2}}\left(c_{0}-c_{+}\right) K_{1}\left(2 e^{-\frac{x}{2}}\right), \quad \psi_{0}(x)=4 e^{-\frac{x}{2}}\left(c_{+}-c_{0}\right) K_{0}\left(2 e^{-\frac{x}{2}}\right) .
$$




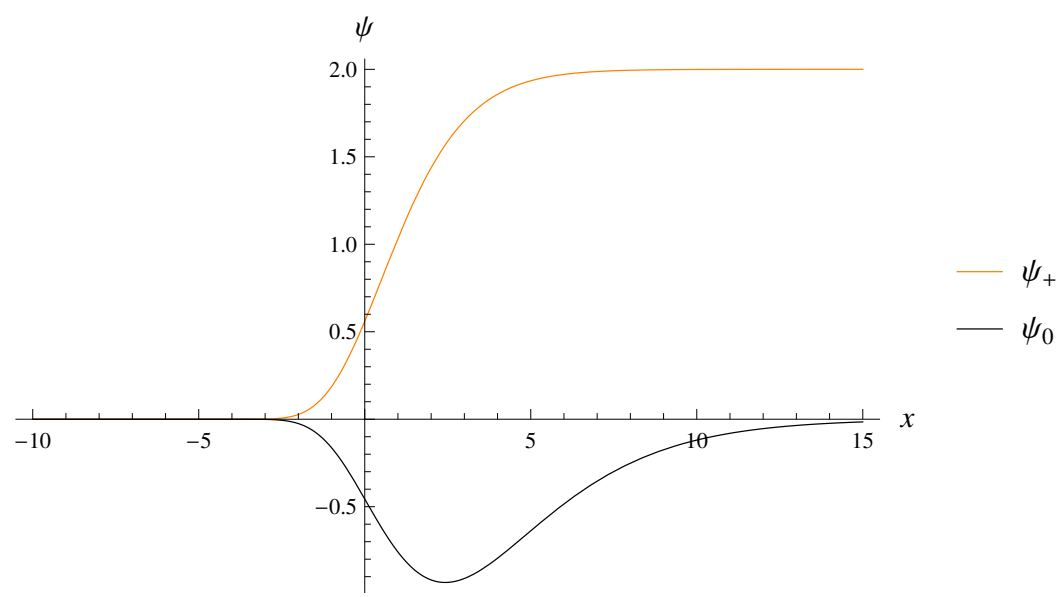

Figure 6. The exact wave function for the spin-1 problem analytically continued to $p_{1}=0$.

As shown in figure $6, \psi_{0}$ is a bound state. Although $\psi_{+}$is not normalizable, it asymptotes to a constant so its momentum is localized at $p_{1}=0$. It corresponds to an anomalous threshold where the relative separation between the particles stays fixed. The presence of an anomalous threshold is usually associated with a double pole in the S-matrix [64], which is indeed the case here. Other singularities of the S-matrix are simple zeros and simple poles but they have negative energy hence are not physical.

For $s_{A}=2$, the first-order Darboux transform is not sufficient to separate the equations, and one needs to consider the second-order Darboux transform of the form $Q=$ $\left(d+A_{2}\right)\left(d+A_{1}\right)$. However, solving for the unknowns is still nontrivial and the difficulty increases with the order. One may hope to use the bootstrap method to obtain the S-matrix for $s_{A}=2$ from the known S-matrices for lower spins by letting one particle go to its pole. Yet we have shown that the only physical pole for $s_{A}=1$ corresponds to an anomalous threshold so does not form a bound state.

\subsubsection{The compact $\mathfrak{s u}(2)$ model}

Although the direct solution of the matrix Schrödinger problem for general spins is beyond reach for now, the solutions we found for spins $0,1 / 2$ and 1 are sufficient for the fermionic model, where the spins are in the fundamental representation of $\mathfrak{s u}(2)$. There is again an auxiliary $\mathfrak{s u}(2)$ symmetry that hops spins from one site to another. The total number of spins gives us another $\mathfrak{u}(1)_{N}$ that commutes with both the global $\mathfrak{s u}(2)_{G}$ and the auxiliary $\mathfrak{s u}(2)_{A}$ symmetries. Because the vector space is finite-dimensional, we can write out the bases of two-particle states explicitly. They are organized into multiplets carrying charges under the symmetries, as shown in table 3 .

The two-body S-matrix decomposes into total spin $s_{G}=0$ and $s_{G}=1$ components as

$$
\mathbb{S}(p)=S_{s_{G}=0}(p) \mathcal{P}_{0}+S_{s_{G}=1}(p) \mathcal{P}_{1}
$$

We will focus on the case when there are $N=2$ spins between two sites and exactly one spin at each site $\Delta s \equiv N_{1}-N_{2}=0$. This corresponds to having exactly one spin at each 


\begin{tabular}{|c|c|c|c|}
\hline $\mathfrak{u}(1)_{N}$ & $\mathfrak{s u}(2)_{G}$ & $\mathfrak{s u}(2)_{A}$ & States \\
\hline 0 & 0 & 0 & $|0,0\rangle$ \\
\hline 1 & $\frac{1}{2}$ & $\frac{1}{2}$ & $|\uparrow, 0\rangle, \quad|0, \uparrow\rangle, \quad|\downarrow, 0\rangle, \quad|0, \downarrow\rangle$ \\
\hline 2 & 1 & 0 & $|\uparrow, \uparrow\rangle, \quad|\uparrow, \downarrow\rangle+|\downarrow, \uparrow\rangle, \quad|\downarrow, \downarrow\rangle$ \\
\hline 2 & 0 & 1 & $|\uparrow \downarrow, 0\rangle, \quad|\uparrow, \downarrow\rangle-|\downarrow, \uparrow\rangle, \quad|0, \uparrow \downarrow\rangle$ \\
\hline 3 & $\frac{1}{2}$ & $\frac{1}{2}$ & $|\uparrow \downarrow, \uparrow\rangle, \quad|\uparrow, \uparrow \downarrow\rangle, \quad|\uparrow \downarrow, \downarrow\rangle, \quad|\downarrow, \uparrow \downarrow\rangle$ \\
\hline 4 & 0 & 0 & $|\uparrow \downarrow, \uparrow \downarrow\rangle$ \\
\hline
\end{tabular}

Table 3. Bases of two-particle states in the fermionic Hubbard-Toda model.

site and is relevant to spin chains. Note that in the $N=2$ sector, $s_{G}=0$ corresponds to $s_{A}=1$ and vice versa. We may read off each component from the results in the previous section.

$$
\begin{aligned}
\mathbb{S}(p) & =S\left(p ; \Delta s=0, s_{A}=1\right) \mathcal{P}_{0}+S\left(p ; \Delta s=0, s_{A}=0\right) \mathcal{P}_{1} \\
& =\frac{\Gamma(1+i p)}{\Gamma(1-i p)}\left(\frac{p-i \mathbb{P}}{p-i}\right) .
\end{aligned}
$$

The operator in the bracket is nothing other than the fundamental R-matrix for the $\mathfrak{s u}(2)$ spin chain. Diagonalizing it with the quantum inverse scattering method described in the previous section, we obtain a set of Bethe ansatz equations for the fermionic $\mathfrak{s u}(2)$ Hubbard-Toda model

$$
\begin{aligned}
e^{i p_{k} L} & =\prod_{\ell \neq k}^{K} \frac{\Gamma\left(1+i\left(p_{k}-p_{\ell}\right)\right)}{\Gamma\left(1-i\left(p_{k}-p_{\ell}\right)\right)} \prod_{i=1}^{M} \frac{p_{k}-\lambda_{i}-\frac{i}{2}}{p_{k}-\lambda_{i}+\frac{i}{2}} \\
\prod_{k=1}^{K} \frac{\lambda_{i}-p_{k}+\frac{i}{2}}{\lambda_{i}-p_{k}-\frac{i}{2}} & =e^{2 \pi i \tau} \prod_{j \neq i}^{M} \frac{\lambda_{i}-\lambda_{j}+i}{\lambda_{i}-\lambda_{j}-i} .
\end{aligned}
$$

\section{Solution by the Bethe/Gauge correspondence}

The quantum inverse scattering method that we discussed in the previous section allows us to diagonalize the integrable system by finding the Bethe ansatz equations. The method is standard albeit somewhat technical. There is a novel way to obtain the Bethe ansatz equations directly from the corresponding gauge theory discovered by Nekrasov and Shatashvili. The so-called Bethe/Gauge correspondence relates the supersymmetric vacua of $\mathcal{N}=2$ field theories with the eigenstates of the quantum integrable systems.

Consider an $\mathcal{N}=2$ supersymmetric gauge theory in four dimensions with gauge group $G$ of rank $r$. As above, the low-energy physics on the Coulomb branch is determined by a holomorphic curve $\Sigma$ of genus $r$ and a meromorphic differential $\lambda_{\mathrm{SW}}$. Picking a canonical set of basis cycles $\left\{A_{I}, B_{I}\right\}$, with $A_{I} \cap B_{J}=\delta_{I J}$ for $I, J=1, \ldots, r$, we have

$$
\vec{a}=\frac{1}{2 \pi i} \oint_{\vec{A}} \lambda_{\mathrm{SW}}, \quad \vec{a}^{D}=\frac{1}{2 \pi i} \frac{\partial \mathcal{F}}{\partial \vec{a}}=\frac{1}{2 \pi i} \oint_{\vec{B}} \lambda_{\mathrm{SW}}
$$


where $\vec{a}=\left(a_{1}, \ldots, a_{r}\right)$ with similar notation for other $r$-component vectors. These relations determine the prepotential $\mathcal{F}=\mathcal{F}(\vec{a})$ which in turn determines the exact low-energy effective action on the Coulomb branch. As discussed in section 2.2 above, the moduli of the Seiberg-Witten curve $\Sigma$ can be identified with the Poisson-commuting Hamiltonians of a complex classical integrable system. Further the periods of $\lambda_{\mathrm{SW}}$ around half of the basis cycles, for example the cycles $\left\{B_{I}\right\}, I=1, \ldots, r$, correspond to canonical action variables for the complex integrable system. To define a real quantum integrable system we need to choose a middle-dimensional real slice of the Coulomb branch and then quantize the corresponding action variables appropriately. In the Bethe/Gauge correspondence, these two steps are accomplished simultaneously by introducing an $\Omega$-background in one plane of the $4 \mathrm{~d}$ spacetime of the gauge theory. More precisely we consider the corresponding Nekrasov partition function $[65,66]$

$$
\mathcal{Z}\left(\vec{a}, \epsilon_{1}, \epsilon_{2}\right)
$$

with deformation parameters $\epsilon_{1}, \epsilon_{2}$. Taking the limit $\epsilon_{2} \rightarrow 0$ with $\epsilon=\epsilon_{1}$ held fixed, we define a "quantum" prepotential

$$
\mathcal{F}(\vec{a}, \epsilon)=\left.\lim _{\epsilon_{2} \rightarrow 0} \epsilon_{1} \epsilon_{2} \log \mathcal{Z}\left(\vec{a}, \epsilon_{1}, \epsilon_{2}\right)\right|_{\epsilon_{1}=\epsilon} .
$$

which reduces to the prepotential of the undeformed theory in the limit $\epsilon \rightarrow 0$.

In the presence of the $\Omega$-background in one plane, $4 \mathrm{~d}$ Lorentz invariance is broken and one obtains a 2 d effective theory with $\mathcal{N}=(2,2)$ supersymmetry in the orthogonal plane. The supersymmetric vacua of this theory are determined by the stationary points of an effective superpotential

$$
\mathcal{W}(\vec{a}, \epsilon)=\frac{1}{\epsilon} \mathcal{F}(\vec{a}, \epsilon)-2 \pi i \vec{k} \cdot \vec{a},
$$

where the vector of integers, $\vec{k} \in \mathbb{Z}^{r}$ corresponds to a choice of branch for the perturbative logarithms appearing in $\mathcal{F}$. In the following we will suppress the second term in (4.4) and work instead with a multi-valued superpotential. The stationary points of this potential correspond to states in the spectrum of a quantum integrable system where the deformation parameter is identified with Planck's constant as $\epsilon=-i \hbar$. In particular, working to leading order in $\epsilon$ we obtain the quantization condition

$$
\frac{\partial \mathcal{W}}{\partial \vec{a}}=0 \Rightarrow \vec{a}^{D} \in \epsilon \mathbb{Z}^{r}
$$

In particular, setting $\epsilon=-i \hbar$, this relation imposes the condition $\operatorname{Re} \vec{a}^{D}=0$, which picks out a middle-dimensional real slice of the Coulomb branch. Then $\operatorname{Im} \vec{a}^{D}$ correspond to the canonical action variables of the corresponding real integrable system and (4.5) coincides with the Bohr-Sommerfeld quantization condition for this system. Higher-order corrections in $\epsilon$ correct the Bohr-Sommerfeld condition to give an exact quantization of the system. Values of the conserved Hamiltonians in each quantum state are determined by the resulting on-shell values of the superpotential.

An important feature of integrable systems captured by the Bethe/Gauge correspondence is that a single complex integrable system can give rise to several inequivalent real 
integrable systems. In supersymmetric gauge theory this feature is related to the electromagnetic duality group of the low-energy theory on the Coulomb branch which corresponds to the group $\operatorname{Sp}(2 r, \mathbb{Z})$ of modular transformations of the Seiberg-Witten curve $\Sigma$. The basis cycles defined above transform linearly under the action of the modular group, giving a new set of quantization conditions. For example, performing a $\mathbb{Z}_{2}$ electro-magnetic duality transformation, we obtain the dual superpotential

$$
\mathcal{W}_{D}\left(\vec{a}^{D}\right)=\mathcal{W}(\vec{a})+\frac{2 \pi i}{\epsilon} \vec{a} \cdot \vec{a}^{D},
$$

whose F-term equations give rise to dual quantization conditions

$$
\frac{\partial \mathcal{W}_{D}}{\partial \vec{a}^{D}}=0 \Rightarrow \vec{a} \in \epsilon \mathbb{Z}^{r} .
$$

In the classical limit, $\epsilon \rightarrow 0$, these give the reality condition $\operatorname{Re} \vec{a}=0$, which yields a real integrable system inequivalent to the one discussed above. Working at non-zero $\epsilon=-i \hbar$ provides a quantization of this system. In the terminology of [6] this is known as the Bquantization, while the original condition (4.5) corresponds to the A-quantization. More generally, each element of the low-energy duality group yields a distinct quantum integrable system in this way.

We now turn to the integrable systems corresponding to elliptic quiver gauge theories. For simplicity, we will focus on the $N=2$ case, in other words the $\hat{A}_{1}$ quiver with gauge group $G=\mathrm{U}(1) \times \mathrm{SU}(K)_{1} \times \mathrm{SU}(K)_{2}$, whose IIA brane construction is illustrated in figure 1 . As above we have Coulomb branch parameters $a_{k}^{(1)}$ and $a_{k}^{(2)}$ for the two $\mathrm{SU}(K)$ factors in $G$. The corresponding cycles on the Seiberg-Witten curve are $\vec{A}^{(\alpha)}$ for $\alpha=1,2$. We also define complexified couplings $\tau_{\alpha}$ for $\mathrm{SU}(K)_{\alpha}$ and bi-fundamental masses $m_{\alpha}$ for $\alpha=1,2$. For cosmetic reasons, the Coulomb branch parameters $a_{k}^{(1)}$ and $a_{k}^{(2)}$ will be renamed $a_{k}$ and $b_{k}$ respectively for $k=1, \ldots, K$ and will be organized as $K$-component vectors $\vec{a}=$ $\left(a_{1}, \ldots, a_{K}\right), \vec{b}=\left(b_{1}, \ldots, b_{K}\right)$. The solution of the model is then specified by the quantum prepotential $\hat{\mathcal{F}}(\vec{a}, \vec{b}, \epsilon)$.

The $N=2$ case of the $K$-body elliptic spin Calogero-Moser model, considered above as a complex classical integrable system, corresponds to the special strong-coupling point $\tau_{1}=0$ of the $\hat{A}_{1}$ quiver theory. According to the discussion above, to choose a real integrable system and quantize it, we need to select a set of basis cycles for the curve. To begin we change basis to cycles $\vec{A}_{+}=\vec{A}^{(1)}$ and $\vec{A}_{-}=\vec{A}^{(1)}-\vec{A}^{(2)}$, with similar definitions for $\vec{B}_{ \pm}$. The corresponding periods are

$$
\vec{a}_{ \pm}=\frac{1}{2 \pi i} \oint_{\vec{A}_{ \pm}} \lambda_{\mathrm{SW}}, \quad \vec{a}_{ \pm}^{D}=\frac{1}{2 \pi i} \frac{\partial \mathcal{F}}{\partial \vec{a}_{ \pm}}=\frac{1}{2 \pi i} \oint_{\vec{B}_{ \pm}} \lambda_{\mathrm{SW}}
$$

which are related to the original Coulomb branch variables via

$$
\vec{a}=\vec{a}_{+}, \quad \vec{b}=\vec{a}_{+}-\vec{a}_{-} .
$$

We will shortly see that the appropriate semi-classical quantization condition for the real elliptic Calogero-Moser model is

$$
\vec{a}_{+}^{D} \in \epsilon \mathbb{Z}^{K}, \quad \vec{a}_{-} \in \epsilon \mathbb{Z}^{K},
$$


which corresponds to a mixed scheme in which we choose the "A-quantization" for the dynamical variables associated with the diagonal $\mathrm{SU}(K)$ and the "B-quantization" for those associated with the off-diagonal $\mathrm{SU}(K)$. Accordingly we define a multi-valued effective superpotential

$$
\mathcal{W}\left(\vec{a}_{+}, \vec{a}_{-}^{D}\right)=\frac{1}{\epsilon}\left[\mathcal{F}\left(\vec{a}_{+}, \vec{a}_{-}\right)-2 \pi i \vec{a}_{-} \cdot \vec{a}_{-}^{D}\right]
$$

where

$$
\mathcal{F}\left(\vec{a}_{+}, \vec{a}_{-}\right)=\hat{\mathcal{F}}\left(\vec{a}_{+}, \vec{a}_{+}-\vec{a}_{-}, \epsilon\right) .
$$

In principle, the on-shell values of the superpotential (4.11) should provide a quantization of the elliptic spin Calogero-Moser model for all values of the parameters. In this paper, we are primarily interested in the large-volume $\operatorname{limit} \operatorname{Im} \tau \rightarrow \infty$ with $\tau_{1}$ held fixed where we can compare with the solution of the model obtained using the asymptotic Bethe ansatz in the preceding sections. In the quiver gauge theory, this limit has a clear interpretation: as $\operatorname{Im} \tau_{2} \rightarrow \infty$, the factor $\mathrm{SU}(K)_{2}$ in the gauge group is frozen out becoming an $\mathrm{SU}(K)$ flavor symmetry. The resulting theory is an $A_{1}$ quiver, in other words an $\mathrm{SU}(K)_{1}$ gauge theory with $K$ hypermultiplets in the fundamental representation and $K$ hypermultiplets in the anti-fundamental representation. We denote the corresponding hypermultiplet masses $\vec{m}_{\mathrm{F}}$ and $\vec{m}_{\mathrm{AF}}$ respectively. This theory has fixed gauge coupling $\tau_{1}$ and Coulomb branch parameters $\vec{a}=\vec{a}^{(1)}$. In the absence of the $\Omega$-deformation, we can identify the mass parameters of this theory as follows: $\vec{m}_{\mathrm{F}}=\vec{b}$ and $\vec{m}_{\mathrm{AF}}=\vec{b}+\vec{m}$ where $\vec{m}=(m, \ldots, m)$ with $m=m_{1}+m_{2}$ as above. In the following, we will propose that these identifications are corrected slightly for non-zero $\epsilon$ to read

$$
\vec{a}=\vec{a}^{(1)}+\frac{3}{2} \vec{\epsilon}, \quad \vec{m}_{\mathrm{F}}=\vec{b}+\frac{3}{2} \vec{\epsilon}, \quad \vec{m}_{\mathrm{AF}}=\vec{b}+\vec{m}-\frac{1}{2} \vec{\epsilon},
$$

with $\vec{\epsilon}=(\epsilon, \ldots, \epsilon)$.

In the limit $\operatorname{Im} \tau \rightarrow \infty$ the quantum prepotential of the affine quiver theory goes over to that of the linear quiver described in the preceding paragraph with prepotential denoted $\mathcal{F}_{\text {linear }}\left(\vec{a}, \vec{m}_{\mathrm{F}}, \vec{m}_{\mathrm{AF}}\right)$. Working at large but finite $\operatorname{Im} \tau$ corresponds to weakly gauging the $\mathrm{SU}(K)$ flavor symmetry, and we restore the leading weak-coupling dynamics of the $\mathrm{SU}(K)_{2}$ vector multiplet. Thus we have two contributions to the prepotential $\hat{\mathcal{F}}(\vec{a}, \vec{b}, \epsilon)=\hat{\mathcal{F}}_{1}+\hat{\mathcal{F}}_{2}$ where

$$
\hat{\mathcal{F}}_{1}=\mathcal{F}_{\text {linear }}\left(\vec{a}, \vec{m}_{\mathrm{F}}, \vec{m}_{\mathrm{AF}}\right),
$$

with the parameter identifications described above and

$$
\hat{\mathcal{F}}_{2}(\vec{b})=-2 \pi i \tau \sum_{k=1}^{K} \frac{b_{k}^{2}}{2}-\epsilon \sum_{k, \ell=1}^{K} \omega_{\epsilon}\left(b_{k}-b_{\ell}\right),
$$

where $\omega_{\epsilon}$ satisfies $\omega_{\epsilon}^{\prime}(x)=-\log \Gamma(1+x / \epsilon)$. The first term on the right-hand side is a classical contribution while the second corresponds to the one-loop contribution of the $\mathrm{SU}(K)_{2}$ vector multiplet.

Including both the above contributions to $\hat{\mathcal{F}}$ we form the effective superpotential $\mathcal{W}$ using (4.11). According to our chosen quantization scheme, the above superpotential should 
be evaluated on shell at the quantized values

$$
\vec{a}_{-}=\vec{a}-\vec{b}=-\vec{n} \epsilon, \quad \vec{n} \in \mathbb{Z}^{K},
$$

and then stationarized with respect to $\vec{a}_{+}$. In terms of the parameters of the linear quiver, the quantization of $\vec{a}_{-}$corresponds to selecting the values $\vec{a}=\vec{m}_{\mathrm{F}}-\vec{n} \epsilon$.

The gauge theory relevant for describing the spin Calogero-Moser model with unbroken $\mathfrak{s l}(2, \mathbb{R})$ symmetry is the $\hat{A}_{1}$ quiver theory in the limit of infinite coupling for the offdiagonal gauge coupling $\tau_{1} \rightarrow 0$. On the other hand, the Nekrasov partition function for the quiver theory and the corresponding superpotential $\mathcal{W}_{1}$ is defined as a series in powers of the instanton factor $q_{1}=\exp \left(2 \pi i \tau_{1}\right)$ which becomes of order one near this point. Remarkably we can pass to a dual description of the theory which effectively resums the instanton series and allows us to obtain explicit results in the limit $\tau_{1} \rightarrow 0$. The dual description corresponds to the world-sheet theory of vortex strings in the four-dimensional gauge theory. In particular, the results of [23] allow us to evaluate the difference

$$
\Delta \mathcal{F}=\left.\mathcal{F}_{\text {linear }}\left(\vec{a}, \vec{m}_{\mathrm{F}}, \vec{m}_{\mathrm{AF}}\right)\right|_{\vec{a}=\vec{m}_{\mathrm{F}}-\vec{n} \epsilon}-\left.\mathcal{F}_{\text {linear }}\left(\vec{a}, \vec{m}_{\mathrm{F}}, \vec{m}_{\mathrm{AF}}\right)\right|_{\vec{a}=\vec{m}_{\mathrm{F}}}
$$

between the value of the quantum prepotential $\mathcal{F}_{\text {linear }}$ of the linear quiver at the on-shell value $\vec{a}=\vec{m}_{\mathrm{F}}-\vec{n} \epsilon$, where $\vec{n}=\left(n_{1}, \ldots, n_{K}\right)$ is a vector of non-negative integers, and its value at the root of the Higgs branch $\vec{a}=\vec{m}_{\mathrm{F}}$. The duality of [23] equates $\Delta \mathcal{F} / \epsilon$ to the on-shell value of the superpotential $\mathcal{W}^{2 \mathrm{~d}}$ of the vortex world-volume theory. The latter is a function of $M$ complex variables $\sigma_{i}$ with $i=1, \ldots, M$ corresponding to the scalars in the twisted chiral multiplets of the $2 \mathrm{~d}$ theory. Explicitly we have [67]

$$
\mathcal{W}^{2 \mathrm{~d}}=2 \pi i \hat{\tau} \sum_{i=1}^{M} \sigma_{i}+\sum_{i, j=1}^{M} f\left(\sigma_{i}-\sigma_{j}-\epsilon\right)+\sum_{i=1}^{M} \sum_{k=1}^{K} f\left(\sigma_{i}-\tilde{M}_{k}\right)-\sum_{i=1}^{M} \sum_{k=1}^{K} f\left(\sigma_{i}-M_{k}\right),
$$

where $f(x)=x \log (x / \epsilon)-x$. The parameters appearing in the superpotential correspond to the masses $\vec{M}_{\mathrm{F}}=\left(M_{1}, \ldots, M_{K}\right)$ and $\vec{M}_{\mathrm{AF}}=\left(\tilde{M}_{1}, \ldots, \tilde{M}_{K}\right)$ of chiral multiplets in the fundamental and the anti-fundamental of the $2 \mathrm{~d}$ gauge group $\mathrm{U}(M)$ and the complexified Fayet-Iliopoulos parameter $\hat{\tau}$ of the vortex theory. These are related to the parameters of the $4 \mathrm{~d}$ linear quiver theory via

$$
\hat{\tau}=\tau+\frac{1}{2}(M+1), \quad \vec{M}_{F}=\vec{m}_{\mathrm{F}}-\frac{3}{2} \vec{\epsilon}, \quad \vec{M}_{A F}=\vec{m}_{\mathrm{AF}}+\frac{1}{2} \vec{\epsilon} .
$$

In order to determine the on-shell value of the $2 \mathrm{~d}$ superpotential we compute its stationary values with respect to the $2 \mathrm{~d}$ fields $\sigma_{i}$, which yields the Bethe ansatz-like equations

$$
\prod_{k=1}^{K} \frac{\sigma_{i}-M_{k}}{\sigma_{i}-\tilde{M}_{k}}=e^{2 \pi i \tau_{1}} \prod_{j \neq i}^{M} \frac{\sigma_{i}-\sigma_{j}-\epsilon}{\sigma_{i}-\sigma_{j}+\epsilon} .
$$

To evaluate the superpotential (4.11) at the on-shell values of $\vec{a}_{-}$, we include all the contributions described above:

$$
\mathcal{W}\left(\vec{a}_{+}\right)=\mathcal{W}^{2 \mathrm{~d}}+\left.\frac{1}{\epsilon} \mathcal{F}_{\text {linear }}\left(\vec{a}, \vec{m}_{\mathrm{F}}, \vec{m}_{\mathrm{AF}}\right)\right|_{\vec{a}=\vec{m}_{\mathrm{F}}}+\frac{1}{\epsilon} \hat{\mathcal{F}}_{2} .
$$


To complete our calculation we need to evaluate the final term which corresponds to the value of the superpotential at the Higgs branch root. Here we will use the fact that the theory at the root reduces to that of the weakly-gauged $\mathrm{SU}(K)_{2}$ vector multiplet coupled to a single adjoint hypermultiplet of mass $m=m_{1}+m_{2}$. The classical prepotential of this theory is already accounted for in $\hat{\mathcal{F}}_{2}$ as is the one-loop contribution of the vector multiplet. The remaining contribution is that of the adjoint hypermultiplet. Thus we must have

$$
\left.\mathcal{F}_{\text {linear }}\left(\vec{a}, \vec{m}_{\mathrm{F}}, \vec{m}_{\mathrm{AF}}\right)\right|_{\vec{a}=\vec{m}_{\mathrm{F}}}=\epsilon \sum_{k, \ell=1}^{K} \omega_{\epsilon}\left(b_{k}-b_{\ell}+m\right) .
$$

This equality can also be understood directly from the IIA string theory construction of the duality of [23].

To obtain the superpotential as a function of $\vec{a}_{+}$, we impose the equations of motion (4.20) for $\sigma_{j}$ and eliminate $\vec{M}_{\mathrm{F}}, \vec{M}_{\mathrm{AF}}$ and $\vec{b}$ in terms of $\vec{a}_{+}$using (4.9), (4.13), (4.16), (4.19). Finally we minimize the resulting superpotential with respect to $\vec{a}_{+}$to obtain

$$
\begin{aligned}
e^{\frac{b_{k}}{\epsilon} 2 \pi i \tau} & =\prod_{\ell=1}^{K} \frac{\Gamma\left(1+\frac{m}{\epsilon}-\frac{b_{k \ell}}{\epsilon}\right) \Gamma\left(1+\frac{b_{k \ell}}{\epsilon}\right)}{\Gamma\left(1+\frac{m}{\epsilon}+\frac{b_{k \ell}}{\epsilon}\right) \Gamma\left(1-\frac{b_{k \ell}}{\epsilon}\right)} \prod_{i=1}^{M} \frac{b_{k}-\sigma_{i}}{b_{k}-\sigma_{i}-m} \\
\prod_{k=1}^{K} \frac{\sigma_{i}-b_{k}}{\sigma_{i}-b_{k}+m} & =e^{2 \pi i \tau_{1}} \prod_{j \neq i}^{M} \frac{\sigma_{i}-\sigma_{j}-\epsilon}{\sigma_{i}-\sigma_{j}+\epsilon}
\end{aligned}
$$

where $b_{k \ell}=b_{k}-b_{\ell}$ and, for on-shell values of $\vec{a}_{-}$we have

$$
\vec{b}=\vec{a}_{+}+\vec{n} \epsilon
$$

Once we identify the twisted chiral scalars $\sigma_{i}$ with the magnon rapidities $\lambda_{i}$ as $\sigma_{i}=$ $\lambda_{i}-m / 2$, these are precisely the Bethe ansatz equations for the spin Calogero-Sutherland model (3.25), (3.26)! Here we identify the Coulomb branch parameter $b_{k}$ with the particle momentum $p_{k}$ and the mass of the adjoint hypermultiplet $m$ with $2 s \epsilon$ where $s \in \mathbb{Z} / 2$ is the spin of the $\mathfrak{s l}(2, \mathbb{R})$ representation at each site. As above we set $\epsilon=-i \hbar$. In fact these equations hold for both $\mathfrak{s u}(2)$ representations with $s>0$ and $\mathfrak{s l}(2, \mathbb{R})$ representations corresponding to $s<0$.

Now we turn to the Hubbard-Toda chain. Again we will focus on the $N=2$ case where the spins lie in lowest-weight representations of $\mathfrak{s l}(2, \mathbb{R})$. To find an exact quantization of this system using the Bethe/Gauge correspondence we will start with the elliptic $\hat{A}_{2}$ quiver with gauge group $G=\mathrm{U}(1) \times \mathrm{SU}(K)_{1} \times \mathrm{SU}(K)_{2} \times \mathrm{SU}(K)_{3}$, whose IIA brane construction is illustrated in figure 3. As above we have Coulomb branch parameters $a_{k}^{(1)}, a_{k}^{(2)}$ and $a_{k}^{(3)}$ for the three $\mathrm{SU}(K)$ factors in $G$ with $k=1, \ldots, K$. The corresponding cycles on the Seiberg-Witten curve are $\vec{A}^{(\alpha)}$ for $\alpha=1,2,3$. We also define complexified couplings $\tau_{\alpha}$ for $\mathrm{SU}(K)_{\alpha}$ and bi-fundamental masses $m_{\alpha}$ for $\alpha=1,2,3$. For cosmetic reasons, the Coulomb branch parameters $a_{k}^{(1)}, a_{k}^{(2)}$ and $a_{k}^{(3)}$ will be renamed $c_{k}, a_{k}$ and $b_{k}$ respectively for $k=1, \ldots, K$ and will be organized as $K$-component vectors $\vec{c}, \vec{b}$ and $\vec{a}$. The solution of the model is then specified by the quantum prepotential $\hat{\mathcal{F}}(\vec{a}, \vec{b}, \vec{c}, \epsilon)$. 
To begin we change basis to cycles

$$
\vec{A}_{+}=\vec{A}^{(1)}, \quad \vec{A}_{-}=\vec{A}^{(2)}-\vec{A}^{(1)}, \quad \vec{A}_{-}^{\prime}=\vec{A}^{(3)}-\vec{A}^{(2)},
$$

with appropriate definitions for the conjugate cycles $\vec{B}_{+}, \vec{B}_{-}$and $\vec{B}_{-}^{\prime}$ which we will not need here. The corresponding periods are

$$
\vec{a}_{+}=\vec{a}, \quad \vec{a}_{-}=\vec{b}-\vec{a}, \quad \vec{a}_{-}^{\prime}=\vec{c}-\vec{b}+\vec{m},
$$

where $\vec{m}=(m, \ldots, m)$ and $m=m_{1}+m_{2}+m_{3}$. The inverse relations are

$$
\vec{a}=\vec{a}_{+}, \quad \vec{b}=\vec{a}_{+}+\vec{a}_{-}, \quad \vec{c}=\vec{a}_{+}+\vec{a}_{-}+\vec{a}_{-}^{\prime}-\vec{m} .
$$

As in the previous example we will use a mixed quantization scheme where the diagonal cycles are treated in the A-quantization and the off-diagonal ones are treated using the Bquantization. Thus the quantization conditions take the form

$$
\vec{a}_{+}^{D} \in \epsilon \mathbb{Z}^{K}, \quad \vec{a}_{-}, \vec{a}_{-}^{\prime} \in \epsilon \mathbb{Z}^{K},
$$

with the corresponding superpotential

$$
\mathcal{W}\left(\vec{a}_{+}, \vec{a}_{-}^{D}, \vec{a}_{-}^{D}\right)=\frac{1}{\epsilon}\left[\mathcal{F}\left(\vec{a}_{+}, \vec{a}_{-}, \vec{a}_{-}^{\prime}\right)-2 \pi i \vec{a}_{-} \cdot \vec{a}_{-}^{D}-2 \pi i \vec{a}_{-}^{\prime} \cdot \vec{a}_{-}^{D}\right]
$$

where $\mathcal{F}\left(\vec{a}_{+}, \vec{a}_{-}, \vec{a}_{-}^{\prime}\right)=\hat{\mathcal{F}}(\vec{a}, \vec{b}, \vec{c}, \epsilon)$ and $\vec{a}, \vec{b}, \vec{c}$ are given by (4.27) above.

As for the spin Calogero-Moser model we will focus on the weak-coupling $\operatorname{limit} \operatorname{Im} \tau \rightarrow$ $\infty$, where $\tau=\tau_{1}+\tau_{2}+\tau_{3}$, holding $\operatorname{Im} \tau_{2}$ fixed. For convenience we also set $\tau_{1}=\tau_{3}=\tau^{\prime}$. In the resulting limit $\operatorname{Im} \tau^{\prime}$ becomes large so that the gauge group factors $\mathrm{SU}(K)_{1}$ and $\mathrm{SU}(K)_{3}$ are weakly coupled. Once again this limit leads to an $A_{1}$ linear quiver with gauge group $\mathrm{SU}(K)_{1}$ where $\mathrm{SU}(K)_{2}$ and $\mathrm{SU}(K)_{3}$ are weakly-gauged flavor symmetries.

In the absence of an $\Omega$-deformation, the Coulomb branch vacuum expectation values of the linear quiver are $\vec{a}=\vec{a}^{(1)}$ while the fundamental and the anti-fundamental hypermultiplets have masses $\vec{m}_{\mathrm{F}}=\vec{b}, \vec{m}_{\mathrm{AF}}=\vec{c}$. In the limit $\operatorname{Im} \tau \rightarrow \infty$, the quantum prepotential of the affine quiver gauge theory goes over to that of the linear quiver denoted $\mathcal{F}_{\text {linear }}\left(\vec{a}, \vec{m}_{\mathrm{F}}, \vec{m}_{\mathrm{AF}}\right)$ plus contributions from the weakly-gauged flavor symmetries which take the form

$$
\mathcal{F}_{\text {weak }}=\hat{\mathcal{F}}_{2}(\vec{b})+\hat{\mathcal{F}}_{2}(\vec{c})+\epsilon \sum_{k, \ell=1}^{K} \omega_{\epsilon}\left(b_{k}-c_{\ell}\right),
$$

where $\hat{\mathcal{F}}_{2}(\vec{b})$, given in $(4.15)$, is the classical and the one-loop vector multiplet contributions for $\mathrm{SU}(K)_{2}$ and $\hat{\mathcal{F}}_{2}(\vec{c})$ is a similar term for $\mathrm{SU}(K)_{3}$. The final term represents the one-loop contribution of the bi-fundamental hypermultiplet of $\mathrm{SU}(K)_{2} \times \mathrm{SU}(K)_{3}$. The resulting superpotential

$$
\mathcal{W}=\frac{1}{\epsilon}\left(\mathcal{F}_{\text {linear }}+\mathcal{F}_{\text {weak }}\right),
$$

should be evaluated at the on-shell values of $\vec{a}_{-}$and $\vec{a}_{-}^{\prime}$ and then stationarized with respect to $\vec{a}_{+}$. 
Once again we can use the duality of [23] to evaluate the contribution of the linear quiver explicitly. Collecting the various contributions to the superpotential and minimizing with respect to $\vec{a}_{+}$yields the following equations

$$
\begin{aligned}
\left(e^{2 \pi i \tau}\right)^{\frac{b_{k}+c_{k}}{2 \epsilon}} & =\prod_{\ell=1}^{K} \frac{\Gamma\left(1+\frac{b_{k \ell}}{\epsilon}\right) \Gamma\left(1+\frac{c_{k \ell}}{\epsilon}\right) \Gamma\left(1+\frac{b_{k}-c_{\ell}}{\epsilon}\right) \Gamma\left(1+\frac{m}{\epsilon}+\frac{c_{k}-b_{\ell}}{\epsilon}\right)}{\Gamma\left(1-\frac{b_{k \ell}}{\epsilon}\right) \Gamma\left(1-\frac{c_{k \ell}}{\epsilon}\right) \Gamma\left(1+\frac{b_{\ell}-c_{k}}{\epsilon}\right) \Gamma\left(1+\frac{m}{\epsilon}+\frac{c_{\ell}-b_{k}}{\epsilon}\right)} \prod_{i=1}^{M} \frac{\sigma_{i}-b_{k}}{\sigma_{i}-c_{k}} \\
\prod_{k=1}^{K} \frac{\sigma_{i}-b_{k}}{\sigma_{i}-c_{k}} & =e^{2 \pi i \tau_{2}} \prod_{j \neq i}^{M} \frac{\sigma_{i}-\sigma_{j}-\epsilon}{\sigma_{i}-\sigma_{j}+\epsilon} .
\end{aligned}
$$

Now we take the Inozemtsev limit $\tau \rightarrow \infty, m \rightarrow \infty$ with $\Lambda=m \exp (\pi i \tau / K)$ held fixed, after which the first equation in (4.32) reduces to

$$
\left(\Lambda^{2 K}\right)^{\frac{b_{k}+c_{k}}{2 \epsilon}}=\prod_{\ell=1}^{K} \frac{\Gamma\left(1+\frac{b_{k \ell}}{\epsilon}\right) \Gamma\left(1+\frac{c_{k \ell}}{\epsilon}\right) \Gamma\left(1+\frac{b_{k}-c_{\ell}}{\epsilon}\right)}{\Gamma\left(1-\frac{b_{k \ell}}{\epsilon}\right) \Gamma\left(1-\frac{c_{k \ell}}{\epsilon}\right) \Gamma\left(1+\frac{b_{\ell}-c_{k}}{\epsilon}\right)} \prod_{i=1}^{M} \frac{\sigma_{i}-b_{k}}{\sigma_{i}-c_{k}} .
$$

We can identify these equations with the Bethe ansatz equations of the Hubbard-Toda chain by setting $b_{k}=p_{k}+s_{k} \epsilon, c_{k}=p_{k}-s_{k} \epsilon$ where $\epsilon=-i \hbar$ and $s_{k} \in \mathbb{Z} / 2$ is the spin label of the $k$-th particle. The $2 \mathrm{~d}$ fields $\sigma_{i}$ are identified with the magnon rapidities $\lambda_{i}$. The resulting equations read

$$
\begin{aligned}
e^{-i L \frac{p_{k}}{\hbar}} & =\prod_{\ell=1}^{K} S_{0}\left(p_{k}-p_{\ell} ; s_{k}, s_{\ell}\right) \prod_{i=1}^{M} \frac{p_{k}-\lambda_{i}-i s_{k} \hbar}{p_{k}-\lambda_{i}+i s_{k} \hbar} \\
\prod_{k=1}^{K} \frac{\lambda_{i}-p_{k}+i s_{k} \hbar}{\lambda_{i}-p_{k}-i s_{k} \hbar} & =e^{2 \pi i \tau_{2}} \prod_{j \neq i}^{M} \frac{\lambda_{i}-\lambda_{j}+i \hbar}{\lambda_{i}-\lambda_{j}-i \hbar}
\end{aligned}
$$

Here we define the effective system size $L=-2 K \log \Lambda$. The central scattering phase is given as

$$
S_{0}\left(p ; s_{1}, s_{2}\right)=\frac{\Gamma\left(1+\Delta s+\frac{p}{\epsilon}\right) \Gamma\left(1-\Delta s+\frac{p}{\epsilon}\right) \Gamma\left(-\bar{s}-\frac{p}{\epsilon}\right)}{\Gamma\left(1+\Delta s-\frac{p}{\epsilon}\right) \Gamma\left(1-\Delta s-\frac{p}{\epsilon}\right) \Gamma\left(-\bar{s}+\frac{p}{\epsilon}\right)},
$$

with $\Delta s=s_{1}-s_{2}$ and $\bar{s}=s_{1}+s_{2}$. This gives a prediction for the scalar part of the S-matrix for the Hubbard-Toda model.

\section{Acknowledgments}

We thank Sungjay Lee for collaboration at an early stage of the project. We are indebted to Alexander Gorsky, Kazuo Hosomichi, Io Kawaguchi, Ivan Kostov, Carlo Meneghelli, Nikita Nekrasov, Vasily Pestun, Didina Serban, Di Wang, Dan Xie, Masahito Yamazaki, Kentaroh Yoshida and Xinyu Zhang for very helpful discussions. 


\section{A The Inozemtsev limit to the Hubbard-Toda model}

We can flow from the Calogero-Moser potential to the Toda potential by taking the Inozemtsev limit [37] (see also [68-70]), i.e., sending the coupling and particle positions to infinity as

$$
x_{k}=X_{k}+k \log \mu^{2}, \quad \Lambda^{K}=\mu^{K} e^{2 \pi i \tau} \text { fixed. }
$$

The Lax matrix for the inhomogeneous spin Calogero-Moser model is (2.9)

$$
L_{k \ell}(z)=\delta_{k \ell}\left[p_{k}+\sum_{\alpha=1}^{N} S_{k k}^{\alpha} \zeta\left(z-z_{\alpha}\right)\right]+\left(1-\delta_{k \ell}\right) \sum_{\alpha=1}^{N} S_{k \ell}^{\alpha} \frac{\sigma\left(x_{k \ell}+z-z_{\alpha}\right)}{\sigma\left(x_{k \ell}\right) \sigma\left(z-z_{\alpha}\right)} e^{x_{k \ell}\left(\psi(z)-\psi\left(z_{\alpha}\right)\right)} .
$$

The Hubbard-Toda model arises from the inhomogeneous spin Calogero-Moser model by setting one inhomogeneity at half-period as $z_{N}=i \pi \tau$ and setting the rest at the origin as $z_{\alpha}=0$ for $\alpha=1, \ldots, N-1$. We further set

$$
Q_{k}^{N}=\tilde{Q}_{k}^{N}=\sqrt{\mu}, \quad \sum_{\alpha=1}^{N-1} S_{k k}^{\alpha}=m-\mu
$$

For this configuration, the Lax matrix becomes

$$
\begin{aligned}
L_{k \ell}(z)= & \delta_{k \ell}\left[p_{k}+\frac{m-\mu N}{N}\left(\zeta(z)-\zeta\left(z-\omega_{2}\right)\right)\right] \\
& +\left(1-\delta_{k \ell}\right) e^{x_{k \ell} \psi(z)}\left[A_{k \ell} \frac{\sigma\left(x_{k \ell}+z\right)}{\sigma\left(x_{k \ell}\right) \sigma(z)}+\mu \frac{\sigma\left(x_{k \ell}+z-\omega_{2}\right)}{\sigma\left(x_{k \ell}\right) \sigma\left(z-\omega_{2}\right)} e^{\zeta\left(\omega_{2}\right) x_{k \ell}}\right] .
\end{aligned}
$$

We first examine the diagonal part. Using the asymptotic formula for $\zeta(z)$ as $\operatorname{Im} \tau \rightarrow$ $\infty$, one can show that in the Inozemtsev limit the diagonal part becomes

$$
L_{k k}(z)=p_{k}+\frac{m-\mu N}{N}\left(\zeta\left(\omega_{2}\right)+\frac{1}{2} \operatorname{coth} \frac{z}{2}\right) .
$$

As we can shift the Lax matrix by a constant times the identity matrix without changing the spectral curve and hence the set of commuting Hamiltonians, we will absorb the siteindependent constant in the diagonal part (A.5) by redefining the $v$ parameter in the spectral curve (2.15).

For the off-diagonal part, the overall factor $e^{x_{k \ell} \psi(z)}$ can also be absorbed by a gauge transformation $L \mapsto g L g^{-1}$ of the diagonal form $g_{k \ell}=\delta_{k \ell} e^{x_{k} \psi(z)}$ that leaves the spectral curve invariant. We use an infinite-series representation [70]

$$
\frac{\sigma\left(x_{k \ell}+z\right)}{\sigma\left(x_{k \ell}\right) \sigma(z)}=e^{\frac{\zeta\left(\omega_{1}\right)}{\omega_{1}} x_{k \ell} z} \sum_{n \in \mathbb{Z}} \frac{e^{n z}}{1-e^{-2 n \omega_{2}-x_{k \ell}}} .
$$

In the $\operatorname{Im} \tau \rightarrow \infty$ limit, the only non-zero contributions are from the $n \leq 0$ terms in the summand. The $n=0$ term tends to 1 if $k>\ell$ and tends to 0 if $k<\ell$. It follows that the right-hand side is equal to $\sum_{n \leq 0} e^{n z}$ when $k>\ell$ and is equal to $\sum_{n<0} e^{n z}$ if $k<\ell$. 
In the variable $t=e^{z}$, the part of the Lax matrix that depends on the spin variables can be written more compactly as

$$
L_{k \ell}^{\mathrm{spin}}(z)=A_{k \ell}\left[\delta_{k \ell} \frac{t+1}{2(t-1)}+\Theta_{k \ell} \frac{t}{t-1}+\Theta_{\ell k} \frac{1}{t-1}\right],
$$

where $\Theta_{k \ell}$ is the discrete Heaviside function taking value 1 for $k>\ell$ and zero otherwise. Using the Legendre relation

$$
\omega_{2} \zeta\left(\omega_{1}\right)-\omega_{1} \zeta\left(\omega_{2}\right)=\frac{i \pi}{2}
$$

and setting $\omega_{1}=i \pi, \omega_{2}=i \pi \tau$, we may write

$$
\frac{\sigma\left(x_{k \ell}+z-\omega_{2}\right)}{\sigma\left(x_{k \ell}\right) \sigma\left(z-\omega_{2}\right)} e^{\zeta\left(\omega_{2}\right) x_{k \ell}}=e^{\frac{\zeta\left(\omega_{1}\right)}{\omega_{1}} x_{k \ell} z} \sum_{n \in \mathbb{Z}} \frac{e^{n z-n \omega_{2}-\frac{x_{k \ell}}{2}}}{1-e^{-2 n \omega_{2}-x_{k \ell}}} .
$$

The dominant term in the Inozemtsev limit is $e^{X_{k}-X_{k-1}}$ for $n=0$ and $\Lambda^{K} t^{ \pm 1} e^{ \pm\left(X_{1}-X_{K}\right)}$ for $n= \pm 1$. For the part of the Lax matrix that depends on the dynamical variables $x_{k}, p_{k}$, the long-range interactions are exponentially suppressed and we obtain the nearest-neighbor interaction with the Toda potential

$$
\begin{aligned}
L_{k \ell}^{\text {Toda }}(z)= & \delta_{k \ell} p_{k}+\delta_{(k-1) \ell} e^{\frac{x_{k-1}-X_{k}}{2}}+\delta_{(k+1) \ell} e^{\frac{x_{k}-X_{k+1}}{2}} \\
& +\delta_{k 1} \delta_{\ell K} \frac{\Lambda^{K}}{t} e^{\frac{x_{K}-X_{1}}{2}}+\delta_{k K} \delta_{\ell 1} \Lambda^{K} t e^{\frac{X_{K}-X_{1}}{2}} .
\end{aligned}
$$

\section{B Proof of classical integrability}

Because the Hubbard-Toda model arises as a special limit from a classically integrable model, we expect integrability to persist. In this appendix we prove this by showing that the Lax matrices are intertwined by the classical $r$-matrix of the Toda chain. Classical integrability relies on the existence of an $r$-matrix that intertwines the Lax matrix acting on two vector spaces

$$
\{L(z) \stackrel{\otimes}{,} L(w)\}=[r(z, w), L(z) \otimes \mathbb{I}]-\left[r^{*}(w, z), \mathbb{I} \otimes L(w)\right] .
$$

In index notation, $r=r_{k m \ell n} e_{k \ell} \otimes e_{m n}$ and $r^{*}=r_{\ell n k m} e_{k \ell} \otimes e_{m n}$, where $e_{k \ell}$ is a matrix that has 1 in the $(k, \ell)$-th entry and 0 elsewhere. Let us define $r_{12}=r\left(z_{1}, z_{2}\right) \otimes \mathbb{I}, r_{23}=$ $\mathbb{I} \otimes r\left(z_{2}, z_{3}\right)$ and similarly for $r_{13}$. We also require that the $r$-matrices satisfy the classical Yang-Baxter equation

$$
\left[r_{12}, r_{13}\right]+\left[r_{12}, r_{23}\right]+\left[r_{13}, r_{23}\right]=0,
$$

which appears as the semi-classical limit of the quantum Yang-Baxter equation (3.1) if we write $\mathbb{R}(z)=1+\hbar r(z)+\mathcal{O}\left(\hbar^{2}\right)$. One can see that (B.2) implies the Jacobi identity for the tensor product Poisson bracket. It follows from (B.1) that the traces of powers of the Lax 
matrix are in involution:

$$
\begin{aligned}
&\left\{L^{m}(z) \stackrel{\otimes}{,} L^{n}(w)\right\}= \sum_{r=0}^{m-1} \sum_{s=0}^{n-1} L^{r}(z) \otimes L^{s}(w)\{L(z) \stackrel{\otimes}{,} L(w)\} L^{m-r-1}(z) \otimes L^{n-s-1}(w) \\
&= \sum_{r=0}^{m-1} \sum_{s=0}^{n-1} L^{r}(z) \otimes L^{s}(w)\left(\left[r_{12}(z, w), L(z) \otimes \mathbb{I}\right]-\left[r_{21}(w, z), \mathbb{I} \otimes L(w)\right]\right) \\
& \times L^{m-r-1}(z) \otimes L^{n-s-1}(w) .
\end{aligned}
$$

Taking the trace and using the cyclic property, we see that $\operatorname{tr} L^{n}(z)$ defines a one-parameter family of commuting conserved charges.

The $r$-matrix for the Toda chain is [71]

$$
r_{k \ell m n}(z, w)=-\left[\delta_{k \ell} \frac{t+s}{2(t-s)}+\Theta_{k \ell} \frac{t}{t-s}+\Theta_{\ell k} \frac{s}{t-s}\right] \delta_{k n} \delta_{\ell m} \equiv-\Delta_{k \ell}^{\prime}(z, w) \delta_{k n} \delta_{\ell m},
$$

where $t=e^{z}, s=e^{w}$. Since it satisfies $r(z, w)=-r^{*}(w, z)$, we can simplify (B.1) as

$$
\{L(z) \stackrel{\otimes}{,} L(w)\}=[r(z, w), L(z) \otimes \mathbb{I}+\mathbb{I} \otimes L(w)] .
$$

Recall the Hubbard-Toda Lax matrix (2.20)

$$
L_{k \ell}(z)=L_{k \ell}^{\mathrm{Toda}}(z)+L_{k \ell}^{\mathrm{spin}}(z)
$$

with $L_{k \ell}^{\text {spin }}(z)=A_{k \ell} \Delta_{k \ell}(z)$ and

$$
\Delta_{k \ell}(z)=\delta_{k \ell} \frac{t+1}{2(t-1)}+\Theta_{k \ell} \frac{t}{t-1}+\Theta_{\ell k} \frac{1}{t-1} .
$$

We check that $L(z)$ and $r(z, w)$ satisfy (B.5). Because the position and momentum variables $x_{k}, p_{k}$ have trivial Poisson bracket with the spin variables $A_{k \ell}$, and it is known that $L^{\text {Toda }}(z)$ satisfies (B.5) with the Toda $r$-matrix, it suffices to check that $L^{\text {spin }}(z)$ also satisfies (B.5) with the same $r$-matrix. The left-hand side can be written as

$$
\begin{aligned}
\left\{L^{\mathrm{spin}}(z) \stackrel{\otimes}{,} L^{\mathrm{spin}}(w)\right\}_{k \ell m n} & \equiv\left\{L_{k m}^{\mathrm{spin}}(z), L_{\ell n}^{\mathrm{spin}}(w)\right\} \\
& =\left(\delta_{k n} A_{\ell m}-\delta_{\ell m} A_{k n}\right) \Delta_{k m}(z) \Delta_{\ell n}(w) .
\end{aligned}
$$

The right-hand side is (writing $r=r(z, w)$ for short and summing over the primed indices)

$$
\begin{aligned}
& {\left[r(z, w), L^{\mathrm{spin}}(z) \otimes \mathbb{I}+\mathbb{I} \otimes L^{\mathrm{spin}}(w)\right]_{k \ell m n}} \\
& \quad=r_{k \ell m^{\prime} n} L_{m^{\prime} m}^{\mathrm{spin}}(z)+r_{k \ell m m^{\prime}} L_{m^{\prime} n}^{\mathrm{spin}}(w)-L_{k m^{\prime}}^{\mathrm{spin}}(z) r_{m^{\prime} \ell m n}-L_{\ell m^{\prime}}^{\mathrm{spin}}(w) r_{k m^{\prime} m n} \\
& \quad=-\delta_{k n} A_{\ell m}\left(\Delta_{k \ell}^{\prime} \Delta_{\ell m}(z)-\Delta_{k m}^{\prime} \Delta_{\ell m}(w)\right)+\delta_{\ell m} A_{k n}\left(\Delta_{n \ell}^{\prime} \Delta_{k n}(z)-\Delta_{k \ell}^{\prime} \Delta_{k n}(w)\right) .
\end{aligned}
$$

One can show that this matches the left-hand side by expanding out the equation above and rewriting it as a product of $\Delta(z)$ and $\Delta(w)$ using the identity

$$
\frac{t+s}{2(t-s)} \frac{1}{s-1}-\frac{1}{t-s} \frac{t}{t-1}=\frac{t+1}{2(t-1)} \frac{1}{s-1} .
$$


Open Access. This article is distributed under the terms of the Creative Commons Attribution License (CC-BY 4.0), which permits any use, distribution and reproduction in any medium, provided the original author(s) and source are credited.

\section{References}

[1] A. Gorsky, I. Krichever, A. Marshakov, A. Mironov and A. Morozov, Integrability and Seiberg-Witten exact solution, Phys. Lett. B 355 (1995) 466 [hep-th/9505035] [INSPIRE].

[2] E.J. Martinec and N.P. Warner, Integrable systems and supersymmetric gauge theory, Nucl. Phys. B 459 (1996) 97 [hep-th/9509161] [INSPIRE].

[3] R. Donagi and E. Witten, Supersymmetric Yang-Mills theory and integrable systems, Nucl. Phys. B 460 (1996) 299 [hep-th/9510101] [INSPIRE].

[4] N.A. Nekrasov and S.L. Shatashvili, Supersymmetric vacua and Bethe ansatz, Nucl. Phys. Proc. Suppl. 192-193 (2009) 91 [arXiv:0901.4744] [INSPIRE].

[5] N.A. Nekrasov and S.L. Shatashvili, Quantum integrability and supersymmetric vacua, Prog. Theor. Phys. Suppl. 177 (2009) 105 [arXiv: 0901.4748] [inSPIRE].

[6] N.A. Nekrasov and S.L. Shatashvili, Quantization of Integrable Systems and Four Dimensional Gauge Theories, in proceedings of the 16th International Congress on Mathematical Physics (ICMP09), Prague, Czech Republic, 3-8 August 2009 [arXiv:0908.4052] [INSPIRE].

[7] N.A. Nekrasov and S.L. Shatashvili, Bethe/Gauge correspondence on curved spaces, JHEP 01 (2015) 100 [arXiv: 1405.6046] [INSPIRE].

[8] D. Orlando and S. Reffert, The Gauge-Bethe Correspondence and Geometric Representation Theory, Lett. Math. Phys. 98 (2011) 289 [arXiv:1011.6120] [InSPIRE].

[9] S. Hellerman, D. Orlando and S. Reffert, String theory of the Omega deformation, JHEP 01 (2012) 148 [arXiv:1106. 0279] [InSPIRE].

[10] D. Orlando and S. Reffert, Twisted Masses and Enhanced Symmetries: the A\&BD Series, JHEP 02 (2012) 060 [arXiv:1111.4811] [INSPIRE].

[11] S. Hellerman, D. Orlando and S. Reffert, The omega deformation from string and M-theory, JHEP 07 (2012) 061 [arXiv:1204.4192] [INSPIRE].

[12] K. Muneyuki, T.-S. Tai, N. Yonezawa and R. Yoshioka, Baxter's T-Q equation, $\mathrm{SU}(N) / \mathrm{SU}(2)^{N-3}$ correspondence and $\Omega$-deformed Seiberg-Witten prepotential, JHEP 09 (2011) 125 [arXiv:1107.3756] [INSPIRE].

[13] A. Mironov, A. Morozov, Y. Zenkevich and A. Zotov, Spectral Duality in Integrable Systems from AGT Conjecture, JETP Lett. 97 (2013) 45 [Pisma Zh. Eksp. Teor. Fiz. 97 (2013) 49] [arXiv: 1204.0913] [INSPIRE].

[14] A. Gadde, S. Gukov and P. Putrov, Walls, Lines and Spectral Dualities in 3d Gauge Theories, JHEP 05 (2014) 047 [arXiv:1302.0015] [INSPIRE].

[15] K. Bulycheva, H.-Y. Chen, A. Gorsky and P. Koroteev, BPS states in omega background and integrability, JHEP 10 (2012) 116 [arXiv:1207.0460] [INSPIRE].

[16] H.-Y. Chen, P.-S. Hsin and P. Koroteev, On the Integrability of Four Dimensional $\mathcal{N}=2$ Gauge Theories in the omega Background, JHEP 08 (2013) 076 [arXiv:1305.5614] [INSPIRE]. 
[17] Y. Luo, M.-C. Tan and J. Yagi, $\mathcal{N}=2$ supersymmetric gauge theories and quantum integrable systems, JHEP 03 (2014) 090 [arXiv:1310.0827] [INSPIRE].

[18] G. Bonelli, A. Sciarappa, A. Tanzini and P. Vasko, Six-dimensional supersymmetric gauge theories, quantum cohomology of instanton moduli spaces and $g l(N)$ Quantum Intermediate Long Wave Hydrodynamics, JHEP 07 (2014) 141 [arXiv:1403.6454] [INSPIRE].

[19] G. Bonelli, A. Sciarappa, A. Tanzini and P. Vasko, Quantum Cohomology and Quantum Hydrodynamics from Supersymmetric Quiver Gauge Theories, J. Geom. Phys. 109 (2016) 3 [arXiv: 1505.07116] [INSPIRE].

[20] P. Koroteev and A. Sciarappa, Quantum Hydrodynamics from Large- $N$ Supersymmetric Gauge Theories, arXiv:1510.00972 [INSPIRE].

[21] J.-E. Bourgine, Y. Matsuo and H. Zhang, Holomorphic field realization of $S H^{c}$ and quantum geometry of quiver gauge theories, JHEP 04 (2016) 167 [arXiv:1512.02492] [INSPIRE].

[22] J. Lamers, The Bethe/Gauge Correspondence, A mysterious link between quantum integrability and supersymmetric gauge theory, MSc Thesis, Utrecht University, Utrecht The Netherlands (2012) http://dspace.library.uu.nl/handle/1874/253835.

[23] N. Dorey, S. Lee and T.J. Hollowood, Quantization of Integrable Systems and a $2 d / 4 d$ Duality, JHEP 10 (2011) 077 [arXiv:1103.5726] [INSPIRE].

[24] H.-Y. Chen, N. Dorey, T.J. Hollowood and S. Lee, A New 2d/4d Duality via Integrability, JHEP 09 (2011) 040 [arXiv:1104.3021] [INSPIRE].

[25] H.-Y. Chen, T.J. Hollowood and P. Zhao, A 5d/3d duality from relativistic integrable system, JHEP 07 (2012) 139 [arXiv: 1205.4230] [INSPIRE].

[26] F. Nieri, S. Pasquetti, F. Passerini and A. Torrielli, 5D partition functions, $q$-Virasoro systems and integrable spin-chains, JHEP 12 (2014) 040 [arXiv:1312.1294] [INSPIRE].

[27] M. Bullimore, H.-C. Kim and P. Koroteev, Defects and Quantum Seiberg-Witten Geometry, JHEP 05 (2015) 095 [arXiv: 1412.6081] [INSPIRE].

[28] D. Orlando and S. Reffert, Relating Gauge Theories via Gauge/Bethe Correspondence, JHEP 10 (2010) 071 [arXiv: 1005.4445] [INSPIRE].

[29] F. Benini, D.S. Park and P. Zhao, Cluster Algebras from Dualities of $2 d \mathcal{N}=(2,2)$ Quiver Gauge Theories, Commun. Math. Phys. 340 (2015) 47 [arXiv:1406.2699] [InSPIRE].

[30] D. Gaiotto and P. Koroteev, On Three Dimensional Quiver Gauge Theories and Integrability, JHEP 05 (2013) 126 [arXiv: 1304.0779] [INSPIRE].

[31] K.K. Kozlowski and J. Teschner, TBA for the Toda chain, in proceedings of the Infinite Analysis 09. New Trends in Quantum Integrable Systems, Kyoto, Japan, 27-31 July 2009, B. Feigin, M. Jimbo and M. Okado eds., World Scientific Publishing Co. Pte. Ltd. (2011), pp. 195-219 [ISBN: 978-981-4324-36-6] [arXiv: 1006.2906] [INSPIRE].

[32] C. Meneghelli and G. Yang, Mayer-Cluster Expansion of Instanton Partition Functions and Thermodynamic Bethe Ansatz, JHEP 05 (2014) 112 [arXiv:1312.4537] [INSPIRE].

[33] J.-E. Bourgine, Confinement and Mayer cluster expansions, Int. J. Mod. Phys. A 29 (2014) 1450077 [arXiv: 1402.1626] [INSPIRE].

[34] Y. Hatsuda and M. Mariño, Exact quantization conditions for the relativistic Toda lattice, JHEP 05 (2016) 133 [arXiv:1511.02860] [INSPIRE]. 
[35] N.A. Nekrasov and V. Pestun, Seiberg-Witten geometry of four dimensional $\mathcal{N}=2$ quiver gauge theories, arXiv:1211.2240 [INSPIRE].

[36] N.A. Nekrasov, V. Pestun and S. Shatashvili, Quantum geometry and quiver gauge theories, arXiv:1312.6689 [INSPIRE].

[37] V.I. Inozemtsev, The Finite Toda Lattices, Commun. Math. Phys. 121 (1989) 629.

[38] E. Witten, Solutions of four-dimensional field theories via M-theory, Nucl. Phys. B 500 (1997) 3 [hep-th/9703166] [INSPIRE].

[39] A. Hanany and D. Tong, Vortices, instantons and branes, JHEP 07 (2003) 037 [hep-th/0306150] [INSPIRE].

[40] A. Hanany and D. Tong, Vortex strings and four-dimensional gauge dynamics, JHEP 04 (2004) 066 [hep-th/0403158] [INSPIRE].

[41] A. Hanany and K. Hori, Branes and $\mathcal{N}=2$ theories in two-dimensions, Nucl. Phys. B 513 (1998) 119 [hep-th/9707192] [INSPIRE].

[42] N. Dorey, The BPS spectra of two-dimensional supersymmetric gauge theories with twisted mass terms, JHEP 11 (1998) 005 [hep-th/9806056] [INSPIRE].

[43] N. Dorey, T.J. Hollowood and D. Tong, The BPS spectra of gauge theories in two-dimensions and four-dimensions, JHEP 05 (1999) 006 [hep-th/9902134] [INSPIRE].

[44] N. Seiberg and E. Witten, Gauge dynamics and compactification to three-dimensions, in proceedings of the Conference on the Mathematical Beauty of Physics (In Memory of C. Itzykson), Saclay, France, 5-7 June 1996 [hep-th/9607163] [InSPIRE].

[45] A. Kapustin, Solution of $\mathcal{N}=2$ gauge theories via compactification to three-dimensions, Nucl. Phys. B 534 (1998) 531 [hep-th/9804069] [INSPIRE].

[46] A. Kapustin and S. Sethi, The Higgs branch of impurity theories, Adv. Theor. Math. Phys. 2 (1998) 571 [hep-th/9804027] [INSPIRE].

[47] K.A. Intriligator and N. Seiberg, Mirror symmetry in three-dimensional gauge theories, Phys. Lett. B 387 (1996) 513 [hep-th/9607207] [INSPIRE].

[48] N. Dorey and A. Singleton, Instantons, Integrability and Discrete Light-Cone Quantisation, arXiv: 1412.5178 [INSPIRE].

[49] N.A. Nekrasov, Holomorphic bundles and many body systems, Commun. Math. Phys. 180 (1996) 587 [hep-th/9503157] [INSPIRE].

[50] N. Dorey, T.J. Hollowood and S.P. Kumar, An Exact elliptic superpotential for $\mathcal{N}=1^{*}$ deformations of finite $\mathcal{N}=2$ gauge theories, Nucl. Phys. B 624 (2002) 95 [hep-th/0108221] [INSPIRE].

[51] J. Gibbons and T. Hermsen, A generalisation of the Calogero-Moser system, Physica D 11 (1984) 337.

[52] J.A. Minahan and A.P. Polychronakos, Integrable systems for particles with internal degrees of freedom, Phys. Lett. B 302 (1993) 265 [hep-th/9206046] [INSPIRE].

[53] Z.N.C. Ha and F.D.M. Haldane, Models with inverse-square exchange, Phys. Rev. B 46 (1992) 9359 [INSPIRE].

[54] I. Krichever, O. Babelon, E. Billey and M. Talon, Spin generalization of the Calogero-Moser system and the matrix KP equation, Amer. Math. Soc. Transl. Ser. 2170 (1995) 92 [hep-th/9411160] [INSPIRE]. 
[55] L.D. Faddeev, E.K. Sklyanin and L.A. Takhtajan, The Quantum Inverse Problem Method. 1, Theor. Math. Phys. 40 (1980) 688 [Teor. Mat. Fiz. 40 (1979) 194] [InSPIRE].

[56] V.E. Korepin, N.M. Bogoliubov and A.G. Izergin, Quantum inverse scattering method and correlation functions, Cambridge University Press (1997).

[57] K. Hikami and M. Wadati, Integrability of Calogero-Moser spin system, J. Phys. Soc. Jpn. 62 (1993) 469 [INSPIRE].

[58] M.A. Olshanetsky and A.M. Perelomov, Classical integrable finite dimensional systems related to Lie algebras, Phys. Rept. 71 (1981) 313 [INSPIRE].

[59] P.P. Kulish, N.Y. Reshetikhin and E.K. Sklyanin, Yang-Baxter Equation and Representation Theory. 1., Lett. Math. Phys. 5 (1981) 393 [InSPIRE].

[60] L.D. Faddeev, Algebraic aspects of Bethe Ansatz, Int. J. Mod. Phys. A 10 (1995) 1845 [hep-th/9404013] [INSPIRE].

[61] L.D. Faddeev, How algebraic Bethe ansatz works for integrable model, in proceedings of the Les Houches School of Physics: Astrophysical Sources of Gravitational Radiation, Les Houches, France, 26 September-6 October 1995, pp. 149-219 [hep-th/9605187] [INSPIRE].

[62] M. Humi, Separation of coupled systems of differential equations by Darboux transformations, J. Phys. A 18 (1985) 1085.

[63] F. Cannata and M.V. Ioffe, Coupled-channel scattering and separation of coupled differential equations by generalized Darboux transformations, J. Phys. A 26 (1993) L89 [INSPIRE].

[64] S.R. Coleman and H.J. Thun, On the Prosaic Origin of the Double Poles in the sine-Gordon S-Matrix, Commun. Math. Phys. 61 (1978) 31 [InSPIRE].

[65] N.A. Nekrasov, Seiberg-Witten prepotential from instanton counting, Adv. Theor. Math. Phys. 7 (2003) 831 [hep-th/0206161] [INSPIRE].

[66] N.A. Nekrasov and A. Okounkov, Seiberg-Witten theory and random partitions, Prog. Math. 244 (2006) 525 [hep-th/0306238] [INSPIRE].

[67] E. Witten, Phases of $\mathcal{N}=2$ theories in two-dimensions, Nucl. Phys. B 403 (1993) 159 [hep-th/9301042] [INSPIRE].

[68] E. D'Hoker and D.H. Phong, Calogero-Moser and Toda systems for twisted and untwisted affine Lie algebras, Nucl. Phys. B 530 (1998) 611 [hep-th/9804125] [INSPIRE].

[69] S.P. Khastgir, R. Sasaki and K. Takasaki, Calogero-Moser models. 4. Limits to Toda theory, Prog. Theor. Phys. 102 (1999) 749 [hep-th/9907102] [INSPIRE].

[70] Y. Chernyakov and A. Zotov, Integrable many body systems via Inosemtsev limit, Theor. Math. Phys. 129 (2001) 1526 [hep-th/0102069] [INSPIRE].

[71] M. Jimbo, Quantum R matrix for the generalized Toda system, Commun. Math. Phys. 102 (1986) 537 [INSPIRE]. 